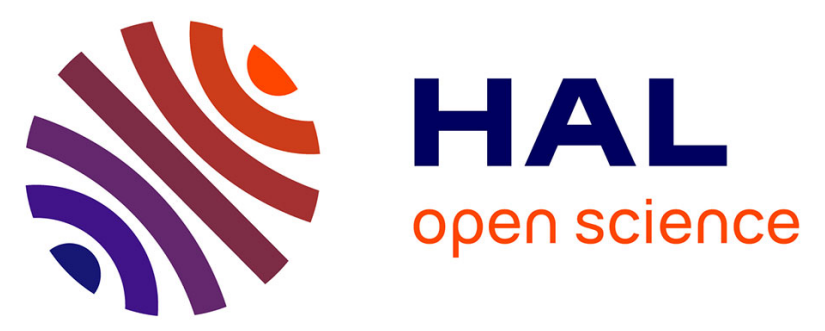

\title{
A new topological approach for the mean field modeling of dynamic recrystallization
}

\author{
Ludovic Maire, Julien Fausty, Marc Bernacki, Nathalie Bozzolo, Pascal de \\ Micheli, Charbel Moussa
}

\section{- To cite this version:}

Ludovic Maire, Julien Fausty, Marc Bernacki, Nathalie Bozzolo, Pascal de Micheli, et al.. A new topological approach for the mean field modeling of dynamic recrystallization. Materials \& Design, 2018, 146, pp.194-207. 10.1016/j.matdes.2018.03.011 . hal-01731431

HAL Id: hal-01731431

https://hal-mines-paristech.archives-ouvertes.fr/hal-01731431

Submitted on 9 May 2018

HAL is a multi-disciplinary open access archive for the deposit and dissemination of scientific research documents, whether they are published or not. The documents may come from teaching and research institutions in France or abroad, or from public or private research centers.
L'archive ouverte pluridisciplinaire HAL, est destinée au dépôt et à la diffusion de documents scientifiques de niveau recherche, publiés ou non, émanant des établissements d'enseignement et de recherche français ou étrangers, des laboratoires publics ou privés. 


\title{
A new topological approach for the mean field modeling of dynamic recrystallization
}

\author{
Ludovic Mairea,*, Julien Fausty ${ }^{\mathrm{a}}$, Marc Bernacki ${ }^{\mathrm{a}}$, Nathalie Bozzolo ${ }^{\mathrm{a}}$, Pascal De \\ Micheli $^{\mathrm{b}}$, Charbel Moussa ${ }^{\mathrm{a}}$ \\ ${ }^{a}$ MINES ParisTech, PSL - Research University, CEMEF - Centre de mise en forme des matriaux, CNRS \\ UMR 7635, CS 10207 rue Claude Daunesse 06904 Sophia Antipolis Cedex, France. \\ ${ }^{b}$ Transvalor S.A., 694 avenue Maurice Donat, 06250 Mougins, France
}

\begin{abstract}
This paper proposes a new approach for mean field modeling of dynamic recrystallization. The main advantage of the presented model compared to the state of the art is based on a more precise description of the immediate vicinity and of the shape of each grain to describe microstructural evolution all along the hot deformation process. Results provided by the new model are compared to those of a former mean field formulation and those of a full field model with an explicit description of the microstructure. The predictions of the new model in terms of recrystallization kinetics and grain size distributions are satisfactory and the progress when compared to former mean field models is obvious. Furthermore, the limitation of mean field models concerning the non-realistic shape of grain size distributions has been solved in this new formulation. Keywords: microstructure, mean field modeling, topology, recrystallization.
\end{abstract}

\section{Introduction}

During hot deformation of metal alloys, the mechanisms of strain hardening and recovery tend to increase and reduce the energy stored in the material respectively. When the stored energy level is high enough locally, new grains nucleate. In parallel, 5 grain boundaries migrate as a result of stored energy gradients across interfaces and capillary effects. The combination of those mechanisms leads to so-called dynamic

\footnotetext{
* Corresponding author

Email address: ludovic.maire@mines-paristech.fr (Ludovic Maire)
} 
recrystallization (DRX) [1, 2, 3, 4, 5].

Over the past decades, much attention has been paid to microstructure evolutions of metal alloys by DRX. These works have lead to different phenomenological models such as those based on the well-known Johnson-Mehl-Avrami-Kolmogorov (JMAK) equations [6, 7, 8], aiming to predict the recrystallized fraction during hot deformation. Considerable progress in terms of numerical resources coupled with new experimental investigation techniques have lead to more sophisticated models able to explicitly reproduce microstructural evolutions: so-called full field methods. The main numerical frameworks for full field modeling of these phenomena are multi phase-field [9], level-set [10], Monte Carlo [11] and cellular automata [12] approaches. These methods have proven to accurately model DRX thanks to an explicit representation of the microstructure. However, a major limitation of these models is their computational cost, 20 especially when crystal plasticity is implemented or when aiming at a direct coupling with macroscale computations.

Mean field models can be proposed as a compromise between the phenomenological laws and full field models. Mean field models for DRX are based on an implicit description of the microstructure by considering grains as spherical entities with an equivalent grain radius and an average dislocation density. Each grain is considered in a homogeneous equivalent medium (HEM) and its evolution is governed by its interaction with the HEM. Hillert [13] proposed the first model of this kind for grain growth. Then, Montheillet et al. [14] proposed a semi-analytical mean field model ture. Another DRX mean field model was also proposed by Cram et al. [15] where the HEM is still composed of all other grains in the microstructure. A first particularity of this model is that each grain is defined by a Taylor/orientation factor aiming to model the influence of the grain orientation on its stress response. Furthermore, mean field 35 models for DRX are generally based on the Taylor assumption, each grain undergoing the same strain. However in the model of Cram et al. [15], each grain undergoes the same mechanical work meaning that softer grains deform more than harder grains. Two 
years after, Bernard et al. [16] proposed a physically-based mean field model whose main novelty lies in a HEM that is subdivided into two different HEMs composed of recrystallized and non-recrystallized grains respectively. Each grain evolves with respect to each of these two HEMs. The main difficulty in this model lies in the fact that the surface fraction between a grain and each of these two HEMs must be estimated at every instant of the simulation. Beltran et al. [17] proposed a model based on that of Bernard [16] where a new nucleation criterion taking dynamic recrystallization into account is implemented. More recently, Piot et al. [18] proposed a new approach in which each grain interacts with a particular HEM composed of one random grain. This model has proven to accurately describe recrystallized fractions and mean grain sizes. Furthermore the predicted grain size distributions are improved as compared to former mean field models [14, 15, 16, 17] even if their are still quite far from experimental ones.

A common advantage to all mean field models is their computational cost that is considerably reduced as compared to full field models. The computational cost of a mean field simulation is generally a few seconds while that of a full field simulations is generally several hours. Furthermore, mean field models generally provide acceptable predictions in terms of recrystallization kinetics and mean grain size evolution. However, grain size distributions sourced from mean field simulations are not correctly described. This limitation is due to the fact that all grains which have nucleated at a given time have the same evolution (in size and dislocation density) in mean field models [14, 15, 16, 17] whereas in a real microstructure, each grain evolves depending on its own neighborhood. Therefore, in this work a new topological approach for the mean field modeling of DRX is proposed. Hereafter, this new model will be called "NeighborHood Model (NHM)". The NHM is based on the same constitutive equations used in the mean field model of Beltran et al. [17] for modeling strain hardening, recovery and nucleation. These laws are introduced in section 2 . The major novelty presented in section 3 is based on the consideration of a particular neighborhood for each grain instead of considering the whole average microstructure as HEM. In addition, the evolution of principal lengths of the grains (in the sense of idealized ellipsoid shapes) are 
modeled during deformation in the NHM, as explained in section 4. Finally, the results of simulations related to hot forging of a $304 \mathrm{~L}$ austenitic steel provided by the NHM, the mean field model of Beltran [17] and a full field level-set model [19] are presented in section 5 .

\section{Constitutive laws for mean field modeling of DRX}

Initial microstructures in mean field models are represented by spherical grains, each having a dislocation density $\rho_{\mathrm{i}}$ and a grain size $R_{\mathrm{i}}$. In several existing models [20, 17], the concept of grain classes is used to reduce computational cost. This consists in gathering several similar grains in one single entity called "class", that is defined by a grain radius $R_{\mathrm{i}}$, a dislocation density $\rho_{\mathrm{i}}$ and a number of grains $N_{\mathrm{i}}$ in the considered class. The main drawback to this reduction is that all the grains belonging to the same class follow the same behaviour during the simulation. This concept of classes is used in the NHM to reduce computational cost. However, in this paper, it will be referred to grains instead of classes in order to facilitate the understanding of the NHM principle.

Thereafter, a recrystallized grain (RX) denotes a grain appeared during the simulation and a non-recrystallized grain (NR) denotes a grain present from the first stage of simulation. Furthermore, a RX grain having just appeared is called nucleus. The constitutive laws used in the NHM are identical to those used in the model of Beltran et al. [17], except for boundary migration. Grain boundary migration is generally described in mean field models [16, 17, 21] by the following equation :

$$
\Delta V_{\mathrm{i}}=\left(K_{3}\left(\rho^{*}-\rho_{\mathrm{i}}\right)+\beta K_{\mathrm{r}}\left(\frac{1}{R^{*}}-\frac{1}{R_{\mathrm{i}}}\right)\right) S_{\mathrm{i}} \Delta t,
$$

where $\Delta V_{\mathrm{i}}$ is the volume variation of the $i^{\text {th }}$ grain, $R_{\mathrm{i}}$ and $\rho_{\mathrm{i}}$ are the radius and the dislocation density of the $i^{\text {th }}$ grain respectively, $R^{*}$ and $\rho^{*}$ are related to the average grain size $\langle R\rangle$ and volume-weighted average dislocation density $\left\langle\rho_{\mathrm{vol}}\right\rangle$ of the HEM respectively. Writing the volume conservation equation $\sum \Delta V_{\mathrm{i}}=0$ leads to $R^{*}=\left\langle R^{2}\right\rangle /\langle R\rangle$ and $\rho^{*}=\left\langle\rho_{\mathrm{vol}}\right\rangle . S_{\mathrm{i}}$ is the boundary area of the $i^{\text {th }}$ grain, $K_{3}$ is a model parameter defined 
by $K_{3}=M_{\mathrm{b}} \tau, K_{\mathrm{r}}$ is a model parameter defined by $K_{\mathrm{r}}=M_{\mathrm{b}} \gamma_{\mathrm{b}}, M_{\mathrm{b}}$ is the grain boundary mobility, $\gamma_{b}$ is the grain boundary energy, $\tau$ is the dislocation line energy and $\beta$ is a geometrical dimensionless constant referring to assumptions on mean field modeling of capillary effects [13], taken equal to 1 in these models [16, 17, 21]. A recent paper has shown that a value of $\beta$ equal to 1.4 leads to more accurate results [22]. $M_{\mathrm{b}}$ and $\gamma_{\mathrm{b}}$ are assumed to be isotropic (i.e. identical for all grain boundaries) in the following. To correctly describe experimental results, it has been shown that $K_{3}$ must be considered dependant on both plastic strain rate $\dot{\varepsilon}$ and temperature $T$ in these models [16, 17, 21]. However, since $K_{3}=M_{\mathrm{b}} \tau$ and there is no clear physical explanation to consider $M_{\mathrm{b}}$ or $\tau$ dependant on the strain rate, an additional term noted $\delta(\dot{\varepsilon})$ is added in $K_{3}$, so that $K_{3}=M_{\mathrm{b}} \tau \delta(\dot{\varepsilon})$. Therefore, Eq. 2 can be rewritten as :

$$
\Delta V_{\mathrm{i}}=M_{\mathrm{b}}\left(\delta(\dot{\varepsilon}) \tau\left(\rho^{*}-\rho_{\mathrm{i}}\right)+\beta \gamma_{\mathrm{b}}\left(\frac{1}{R^{*}}-\frac{1}{R_{\mathrm{i}}}\right)\right) S_{\mathrm{i}} \Delta t .
$$

The evolution of the average dislocation density $\rho_{\mathrm{i}}$ in each $i^{\text {th }}$ grain during deformation is modeled by the Yoshie-Lasraoui-Jonas law [23]:

$$
\frac{\partial \rho_{\mathrm{i}}}{\partial \varepsilon}=K_{1}-K_{2} \rho_{\mathrm{i}}
$$

where $\varepsilon$ denotes the plastic strain, $K_{1}$ and $K_{2}$ are the strain hardening and dynamic recovery terms, respectively, and depend on thermomechanical conditions ( $K_{1}$ depends on $T$ and $K_{2}$ depends on both $T$ and $\dot{\varepsilon}$ ).

When a critical dislocation density is locally reached, nucleation, i.e. appearance of new grains with a very low dislocation density, can occur. To compute the critical 115 dislocation density $\rho_{\mathrm{cr}}$, the method used in [17] is reproduced: a first value of $\rho_{\mathrm{cr}}$ is estimated thanks to Eq. 4 and an iterative calculation is performed according to Eq. 5.

$$
\rho_{\mathrm{cr}}=\left(\frac{20 K_{1} \gamma_{\mathrm{b}} \dot{\varepsilon}}{3 K_{3} \tau}\right)^{1 / 3}
$$




$$
\rho_{\mathrm{cr}}=\left[\frac{-2 \gamma_{\mathrm{b}} \dot{\varepsilon} \frac{K_{2}}{K_{3} \tau}}{\ln \left(1-\frac{K_{2}}{K_{1}} \rho_{\mathrm{cr}}\right)}\right]^{1 / 2} .
$$

The critical radius of a nucleus noted $r^{*}$ is calculated according to the modified BaileyHirsch criterion [24]:

$$
r^{*}=\omega \frac{2 \gamma_{\mathrm{b}}}{\rho_{\mathrm{cr}} \tau},
$$

120 for growth. We consider $\omega=1.5$ in this paper as in [19].

The nucleation rate $\dot{V}$ representing a volume of nuclei per unit of time, is calculated according to a variant of the proportional nucleation model of Peczak and Luton [25]:

$$
\dot{V}=K_{\mathrm{g}} \Phi \Delta t,
$$

125

As it can be observed in Eq. 2, the grain boundary migration of a specific grain depends on the average microstructure through the values $R^{*}$ and $\rho^{*}$, which means that no realistic topology is considered in pre-existing mean field models [14, 15, 16, 17]. An attempt to take a realistic topology into account is presented in section 3 .

\section{Neighborhood of individual grains}

135

In a real microstructure, each grain is surrounded by a number of neighbors (see Fig 1(a)). This microstructure is described in mean field models by spherical grains in order to model equiaxed grains (see Fig. 11(b)), each grain having a radius $R_{\mathrm{i}}$ and 
a dislocation density $\rho_{\mathrm{i}}$. However a strong assumption made in mean field models [14, 15, 17] is that each grain has no neighbors but is surrounded by a HEM (see Fig. 1.(c)). To be more realistic, the new proposed approach tackles this first limitation by considering a particular neighborhood for each grain, composed of a certain number of grains that are appropriately chosen (see Fig. 11(d)).

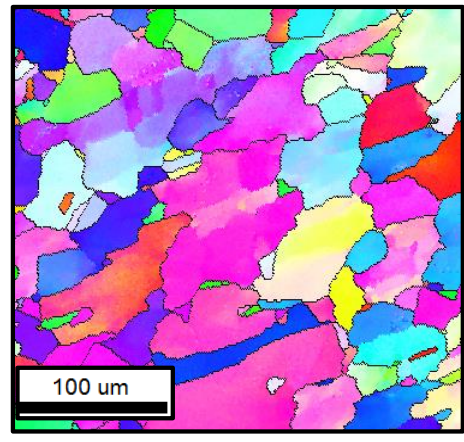

(a)

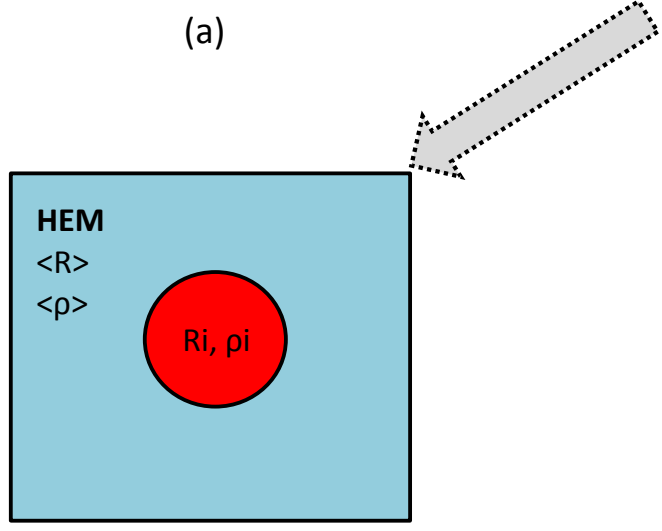

(c)

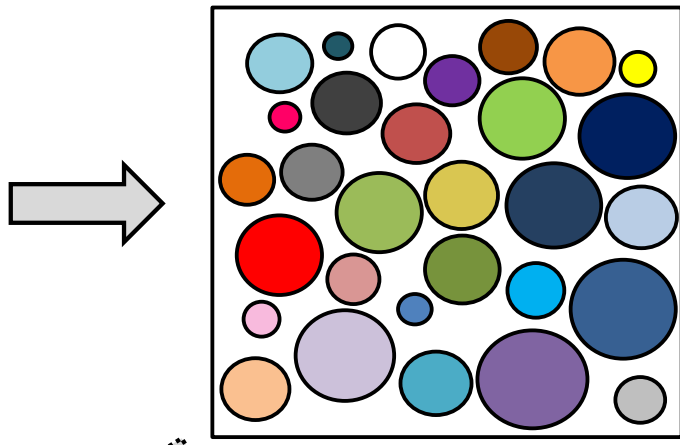

(b)

Figure 1: Representation of the microstructure in mean field models. (a) EBSD picture of an austenitic steel 304L microstructure, (b) representation of a microstructure in mean field models as a set of spherical grains, (c) standard mean field approach : each grain is surrounded by a homogeneous equivalent medium (HEM) composed of all grains in the microstructure and (d) new mean field approach NHM : each grain is surrounded by a certain number of grains that compose its neighborhood.

\subsection{Representation of a grain's neighborhood in the NHM}

In the present approach, necklace nucleation is considered since this is the kind of nucleation observed in 304L during DRX [26, 27]. Thereafter, the quantities $\theta(\mathrm{i})$ and 
(1- $\theta$ )(i) denote the surface fraction of the $i^{\text {th }}$ grain in contact with RX and NR grains respectively. The description of a grain with its neighborhood in the NHM is described in $2 \mathrm{D}$ on Fig. 2. To enrich this description, the quantity $\theta$ (i) is subdivided into two other quantities noted $\theta_{\mathrm{a}}(\mathrm{i})$ and $\theta_{\mathrm{b}}(\mathrm{i})$ which represent the surface fraction of any $i^{\text {th }}$ grain in contact with nuclei and with other RX grains respectively.

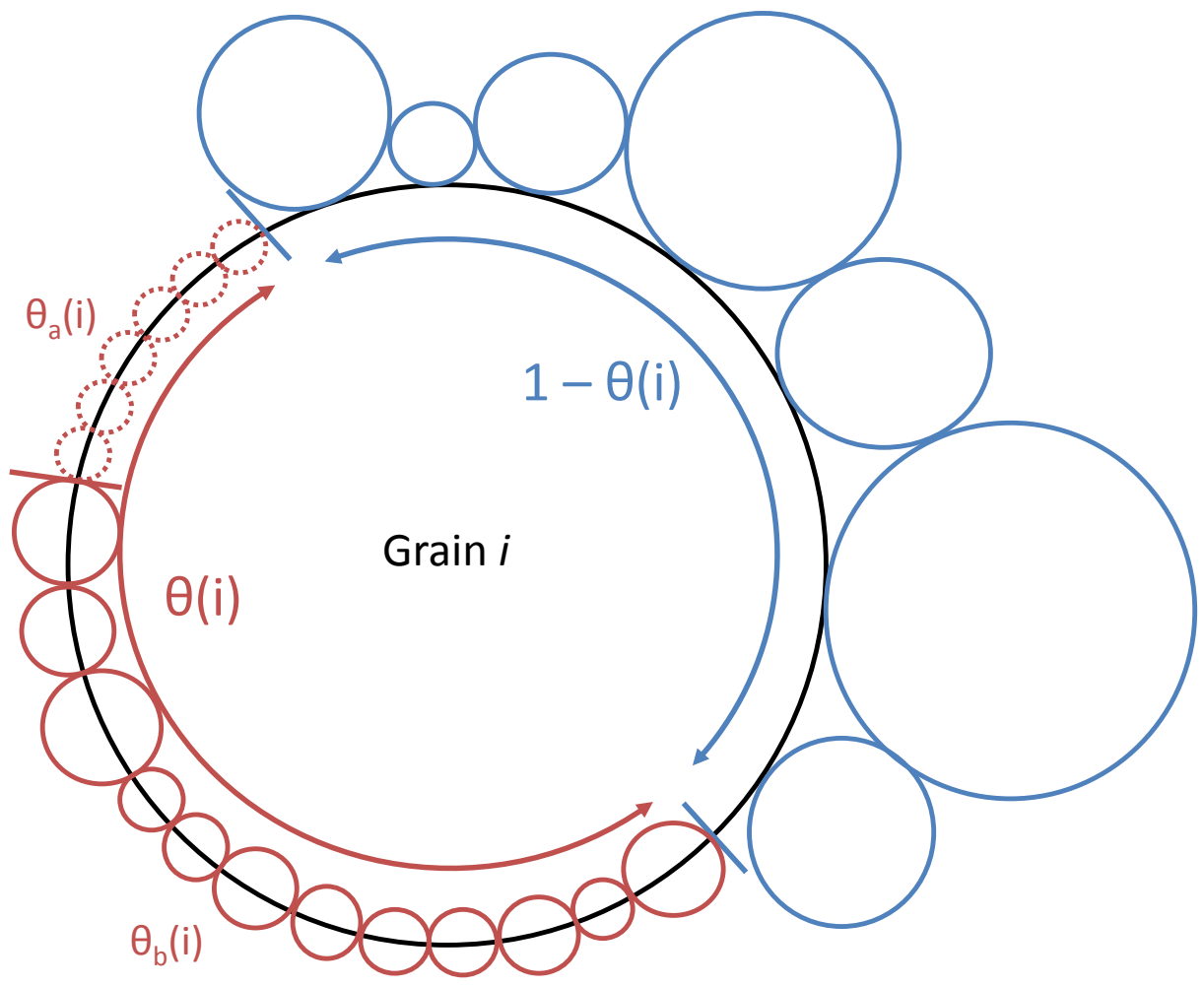

Figure 2: Representation of a grain in the NHM. Each grain $i$ has a surface fraction $\theta(\mathrm{i})$ in contact with recrystallized $(\mathrm{RX})$ grains represented in red colour and a surface fraction $(1-\theta)(\mathrm{i})$ in contact with nonrecrystallized (NR) grains represented in blue colour. $\theta(\mathrm{i})$ is also subdivided into two quantities $\theta_{\mathrm{a}}(\mathrm{i})$ and $\theta_{\mathrm{b}}(\mathrm{i})$ which represent the surface fraction occupied by nuclei in dashed line and other RX grains in full line, respectively.

The 2D schematic surface of a grain $i$ having RX grains appeared on its boundary is represented on Fig. 3 a). The quantity $S 1$ (i) represents the boundary surface between the grain $i$ and RX grains while $S$ 2(i) represents the boundary surface of the grain $i$ that is not in contact with RX grains. In this case, the boundary surface $S_{\mathrm{i}}$ of the grain $i$ 
should be defined by $S_{\mathrm{i}}=S 1(\mathrm{i})+S 2(\mathrm{i})$ and the surface fraction $\theta$ should be defined by $\theta=S 1(\mathrm{i}) /(S 1(\mathrm{i})+S 2(\mathrm{i}))$ on Fig. 3(a). However, in mean field models [14, 15, 17] a surface such as the one presented on Fig. 3. (a) is hard to consider, thus it is common to keep working with the assumption of spherical grains as represented on Fig. 3(b). In the NHM, the assumption of spherical grains presented on Fig. 3(b) is also considered.

160 The quantity S3(i) is defined as the total intersection surface between the considered grain $i$ and RX grains on its boundary (see Fig. 3 b)). Therefore the surface fraction $\theta$ is estimated in the NHM by :

$$
\theta(\mathrm{i}) \simeq\left(\frac{S 3(\mathrm{i})}{S 2(\mathrm{i})+S 3(\mathrm{i})}\right),
$$




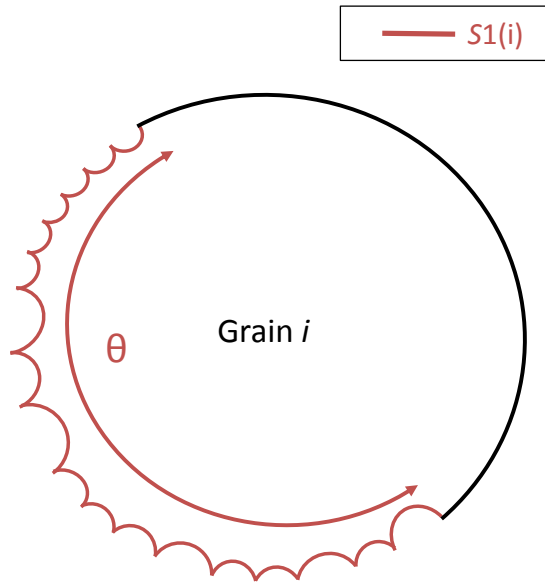

(a)
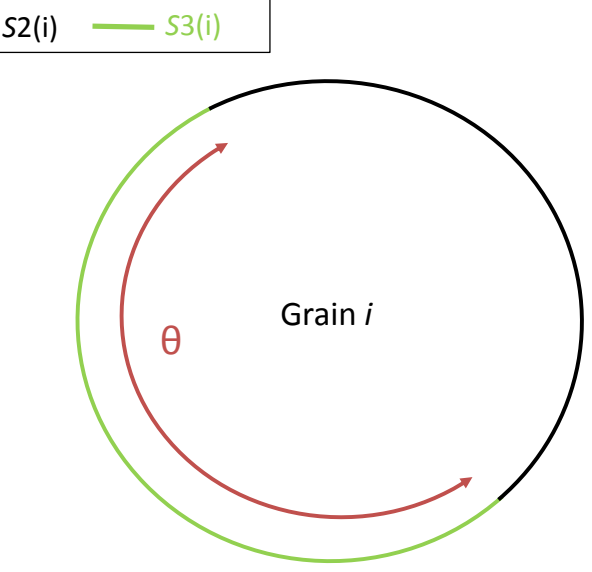

(b)

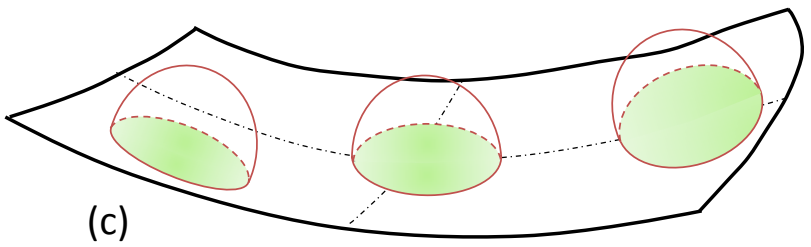

Figure 3: Schematic representation of (a) the surface of the grain $i$ with RX grains on its boundary (b) the surface of the grain $i$ as it is considered in the NHM (c) a 3D portion of a grain boundary with three nuclei as neighbors. The intersections are approximated by several circles in green colour. S1(i) represents the boundary surface between the grain $i$ and RX grains, S2(i) the boundary surface of the grain $i$ that is not in contact with RX grains, S3(i) the total intersection surface between the grain $i$ and RX grains, estimated by a sum of circles in the NHM.

By doing the assumption that the grain $i$ is large as compared to RX grains, every surface of intersection between one RX grain and the grain $i$ are approximated by circles in the NHM as represented on Fig. 3(c). Furthermore by doing the assumption that every recrystallized grain size is approximated by the recrystallized mean grain size $\left\langle R_{\mathrm{rx}}\right\rangle$ with their center belonging to the boundary of $i$, each intersection can be finally defined by a circle of radius $\left\langle R_{\mathrm{rx}}\right\rangle$ having a surface equal to $\pi\left\langle R_{\mathrm{rX}}\right\rangle^{2}$. If $N_{\mathrm{rX}}(\mathrm{i})$ denotes the number of RX grains appeared in the considered grain $i, S 3(\mathrm{i})$ can be estimated by 
$\pi N_{\mathrm{rx}}(\mathrm{i})\left\langle R_{\mathrm{rx}}\right\rangle^{2}$, and $\theta$ can be finally approximated by :

$$
\theta(\mathrm{i}) \simeq\left(\frac{\pi N_{\mathrm{rx}}(\mathrm{i})\left\langle R_{\mathrm{rx}}\right\rangle^{2}}{S_{\mathrm{i}}}\right),
$$

The number of RX grain $N_{\mathrm{rx}}(\mathrm{i})$ appeared in the grain $i$ increases during the simulation while the recrystallized mean grain size $\left\langle R_{\mathrm{rx}}\right\rangle$ and the grain surface $S_{\mathrm{i}}$ are quasiconstant. To avoid values of $\theta(\mathrm{i})$ higher than 1 , the final equation for $\theta(\mathrm{i})$ is defined as:

$$
\theta(\mathrm{i}) \simeq \min \left(\frac{\pi N_{\mathrm{rx}}(\mathrm{i})\left\langle R_{\mathrm{rx}}\right\rangle^{2}}{S_{\mathrm{i}}}, 1\right)
$$

Assuming that Eq. 11 is also available for $\theta_{\mathrm{b}}$, the number of RX neighbors $N_{\theta_{\mathrm{b}}}(\mathrm{i})$ of any $i^{\text {th }}$ grain can be estimated by the following relation :

$$
N_{\theta_{\mathrm{b}}}(\mathrm{i}) \simeq\left(\frac{\theta_{\mathrm{b}}(\mathrm{i}) S_{\mathrm{i}}}{\pi\left\langle R_{\mathrm{rx}}\right\rangle^{2}}\right) .
$$

The number of NR neighbors $N_{(1-\theta)}($ i) must also be estimated. As an approximation, the number of grains on the surface fraction $(1-\theta)(\mathrm{i})$ can be estimated using a law from literature for describing steady state or quasi steady state microstructures. Several kinds of laws have been proposed in the literature to estimate the number of 
and Liu [28] have proposed a linear relationship between the number of neighbors and the mean tangent diameter of grains in 3D microstructures, validated experimentally by Liu et al. [29]. Abbruzese and Campopiano [30] proposed a quadratic relationship between the number of neighbors and the normalized equivalent sphere radius of a grain in 3D microstructures. This relationship has been validated by experimental investigations [29, 31] and numerical studies [32, 33, 34]. All the resulting curves are presented on Fig. 4(b). The curves are globally close to each other. To reinforce this relationship, an additional grain growth numerical simulation has been performed in 3D using a full field method based on a level-set framework (DIGIMU ${ }^{\circledR}$ software package) [35, 19]. An equiaxed microstructure with an average grain size of $100 \mu \mathrm{m}$ has been generated using a Laguerre-Voronoï algorithm [36] and a the simulation of a heat treatment at $1373 \mathrm{~K}$ for $30 \mathrm{~min}$ was performed using model parameters for a $304 \mathrm{~L}$ steel. Grain boundary mobility and energy are considered isotropic in the microstructure. The resulting microstructure is composed of 2000 grains and the number of neighbors of each individual grain is plotted as a function of its normalized equivalent sphere radius on Fig. 4(a) while the average number of neighbors is plotted as a function of the normalized equivalent sphere radius on Fig. 4(b). The curve on Fig. 4(b) resulting from the full field simulation (DIGIMU® software package) is close to the other experimental and numerical curves of the literature, which reinforces the idea that a general polynomial equation can correctly estimate the number of neighbors of grains as a function of their normalized equivalent sphere radius in steady state or quasi steady state 3D microstructures. This polynomial dependence is used in the NHM to estimate the number of neighbors $N_{(1-\theta)}$ (i) at a given stage of the deformation process. This relationship is given in Eq. 14 .

$$
N_{(1-\theta)}(\mathrm{i})=\left(4.06 \times \omega_{\mathrm{i}}^{2}+4.22 \times \omega_{\mathrm{i}}+4.71\right) \times(1-\theta(\mathrm{i}))
$$

where $\omega_{\mathrm{i}}$ is the normalized grain radius defined by $R_{\mathrm{i}} /\left\langle R_{\mathrm{NR}}\right\rangle$. 


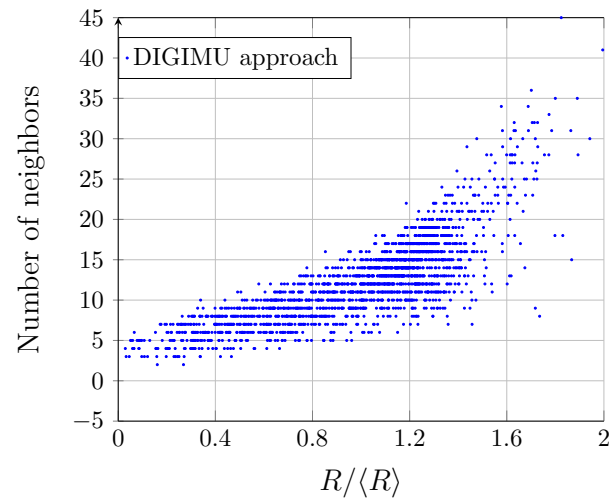

(a)

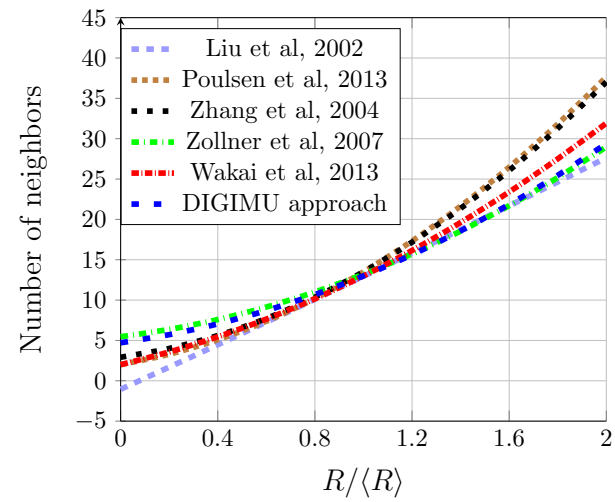

(b)

Figure 4: Number of neighbors as a function of the normalized equivalent sphere radius. All investigations were done on steady or quasi-steady state microstructures. a) Each blue dot represents a grain coming from a full field simulation (DIGIMU ${ }^{\circledR}$ software package) (b) Different curves issued from the literature and from the DIGIMU approach. Those are obtained by least-square regression or by an average per topological classes.

Thus the total number of neighbors of any $i^{\text {th }}$ grain noted $N_{\text {tot }}(\mathrm{i})$ is given by :

$$
N_{\text {tot }}(\mathrm{i})=N_{\theta_{\mathrm{a}}}(\mathrm{i})+N_{\theta_{\mathrm{b}}}(\mathrm{i})+N_{(1-\theta)}(\mathrm{i}) .
$$

\subsection{Criteria for choosing neighbors}

As soon as the quantities $N_{\theta_{\mathrm{b}}}$ (i) and $N_{(1-\theta)}$ (i) have been estimated for any $i^{\text {th }}$ grain thanks to Eqs. 13, 14 respectively, the neighbors are chosen according to the following criteria:

- the neighbors in $N_{\theta_{\mathrm{a}}}(\mathrm{i})$ are the nuclei appeared on the grain boundary of the $\mathrm{i}^{\text {th }}$ grain during the last time step.

- The neighbors in $N_{\theta_{\mathrm{b}}}$ (i) are chosen randomly among all the other RX grains of the microstructure.

- The neighbors in $N_{(1-\theta)}(\mathrm{i})$ are chosen randomly among the NR grains of the microstructure. 
The choice of the neighbors in $N_{\theta_{\mathrm{b}}}(\mathrm{i})$ and $N_{(1-\theta)}(\mathrm{i})$ is made randomly but each grain is weighted by its volume in order to be representative of real microstructures.

A bijectivity is imposed between a grain and its neighbors, meaning that if the $i^{\text {th }}$ grain has the $j^{\text {th }}$ grain as neighbor, the $j^{\text {th }}$ grain has also the $i^{\text {th }}$ grain as neighbor. Thus when the $i^{\text {th }}$ grain grows of a quantity $\mathrm{dV}(\mathrm{i}, \mathrm{j})$ with respect to the $j^{\text {th }}$ grain, the $j^{\text {th }}$ grain shrinks of a quantity $-\mathrm{dV}(\mathrm{i}, \mathrm{j})$ with respect to the $i^{\text {th }}$ grain in order to ensure the bijectivity as well as volume conservation.

It is also worth noticing that the surface fractions $\theta(\mathrm{i})$ and $(1-\theta)(\mathrm{i})$ are only used to identify the respective number of neighbors $\mathrm{N}_{\theta_{\mathrm{b}}}(\mathrm{i})$ and $\mathrm{N}_{(1-\theta)}$ (i). Even so, after the random selection of the neighbors in $\mathrm{N}_{\theta}(\mathrm{i})$ and $\mathrm{N}_{(1-\theta)}(\mathrm{i})$, it can exist a small error on the fractions $\theta(\mathrm{i})$ and $(1-\theta)(\mathrm{i})$. However, this is not a strong assumption since a grain boundary surface is never exactly spherical in reality.

\subsection{Reformulation of boundary migration equation}

Since this new approach considers a particular neighborhood for each grain, Eq. 2 of grain boundary migration presented in the first section has to be reformulated. First, the volume change of the $i^{\text {th }}$ grain is computed according to the following equation in the NHM:

$$
\Delta V_{\mathrm{i}}=\sum_{j=1}^{N_{\mathrm{tot}}(i)} \Delta V_{(\mathrm{i}, \mathrm{j})},
$$

where $\Delta V_{(\mathrm{i}, \mathrm{j})}$ is the volume variation between the $\mathrm{i}^{\text {th }}$ grain and its $\mathrm{j}^{\text {th }}$ neighbors, defined by the equation :

$$
\Delta V_{(\mathrm{i}, \mathrm{j})}=M_{\mathrm{b}}\left(\delta(\dot{\varepsilon}) \tau\left(\rho_{\mathrm{j}}-\rho_{\mathrm{i}}\right)+\beta \gamma_{\mathrm{b}}\left(\frac{1}{R_{\mathrm{j}}}-\frac{1}{R_{\mathrm{i}}}\right)\right) S_{\mathrm{i}} \Psi_{(\mathrm{i}, \mathrm{j})} \Delta t,
$$

where $\rho_{\mathrm{j}}$ and $R_{\mathrm{j}}$ denote the dislocation density and grain radius of a neighbor $j$ of the $i^{\text {th }}$ grain. $\Psi_{(\mathrm{i}, \mathrm{j})}$ is the radius fraction of a grain $j$ with respect to neighbors of $i$, aiming to redistribute the volume variation of the $i^{\text {th }}$ grain on its neighbors depending on their 
size. This quantity is estimated by the following relation:

$$
\Psi_{(\mathrm{i}, \mathrm{j})}=R_{\mathrm{j}} / \sum_{k=1}^{N_{\mathrm{tot}}(i)} R_{k} .
$$

Two flow charts summarizing the main algorithm of NHM as well as the implementation performing boundary migration are presented for a single time step on Fig. 5 5 a) and (b) respectively. 


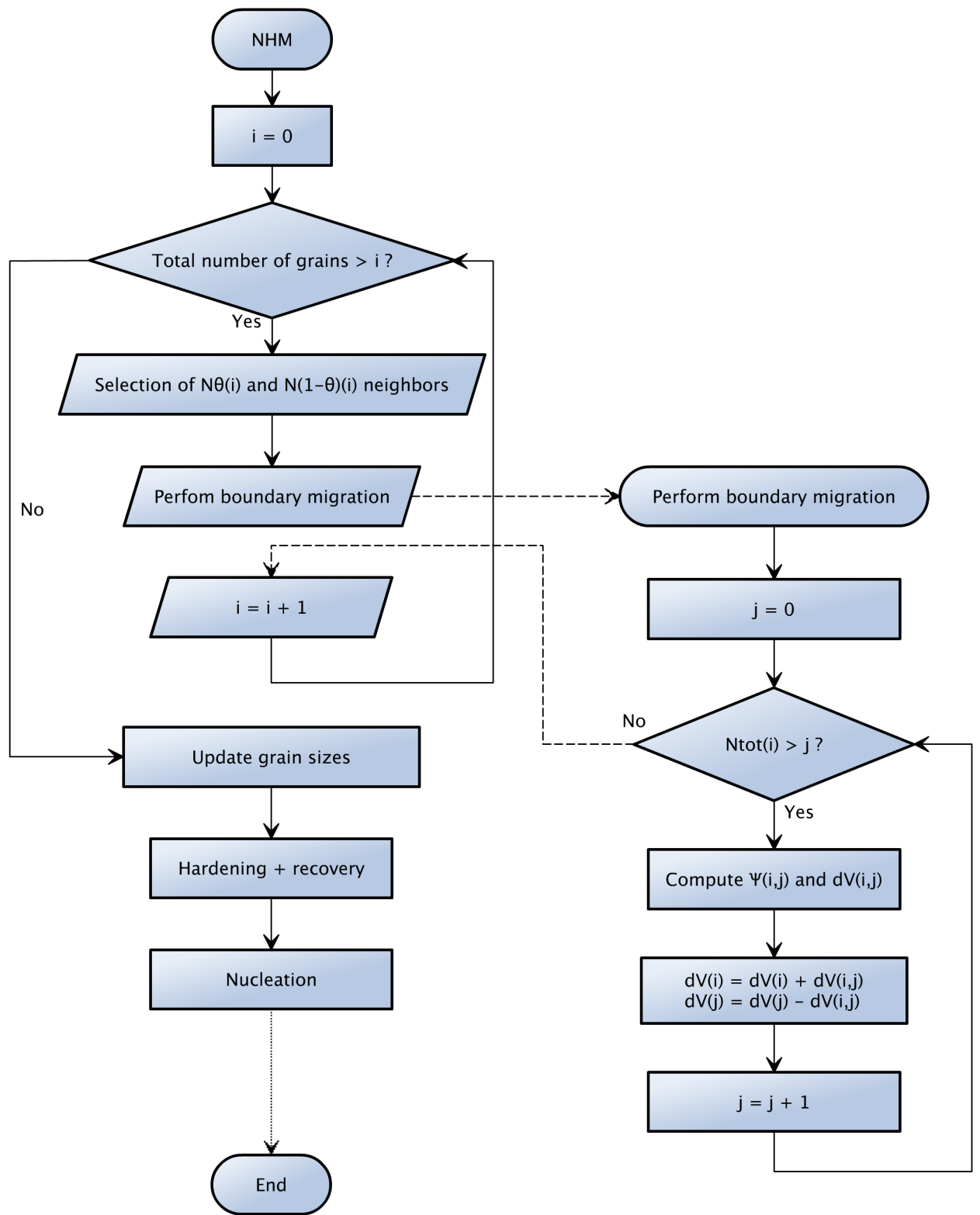

(a)

(b)

Figure 5: Flow charts representing (a) one time step of the main algorithm and (b) the way to perform boundary migration in the NHM. 


\section{Modeling of grain elongation}

To our knowledge, mean field models of the literature [16, 17, 14] assume that the grains remain spherical all along the deformation process. However, it is well-known that the grain shape evolves during deformation. In the case of necklace type nucleation, the assumption of grains remaining spherical all along the deformation process is a strong assumption that can effect the nucleation rate. Indeed, the nucleation rate $\dot{V}$ depends on the grain surface (Eq. 7) which can be underestimated when elongated grains are supposed spherical. Full field models have the advantage of explicitly describing the grain shape evolution during deformation. To investigate the effect of grain shape evolution on the recrystallization kinetics, several full field simulations of DRX have been performed at different strain rates $\left(0.01 \mathrm{~s}^{-1}\right.$ and $\left.0.1 \mathrm{~s}^{-1}\right)$, with and without considering the topological deformation of the representative volume element (RVE) seeing that the dislocation density evolution as well as the nucleation and grain growth mechanisms were still modelled. Two snapshots of the simulations performed at a strain rate of $0.01 \mathrm{~s}^{-1}$ with and without considering the topological deformation of the RVE are presented on Fig. 6. It is worth noting that the simulation presented on Fig. 6.c) makes no physical sense but is only used here to illustrate the potential effect of grain shape evolution on the recrystallization kinetics during DRX with a necklace nucleation. Recrystallized fraction, mean grain size and mean dislocation density are presented as a function of strain on Fig. 7 for the studied strain rates. 


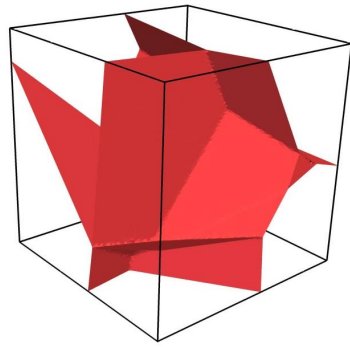

(a) $\varepsilon=0$

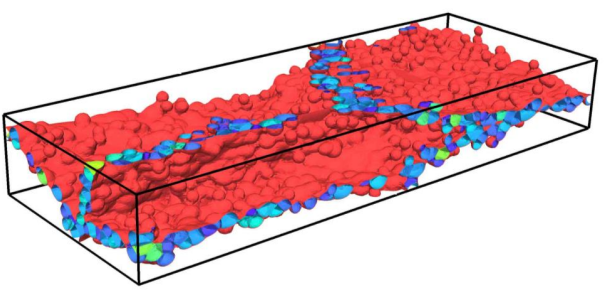

(b) $\varepsilon=1$
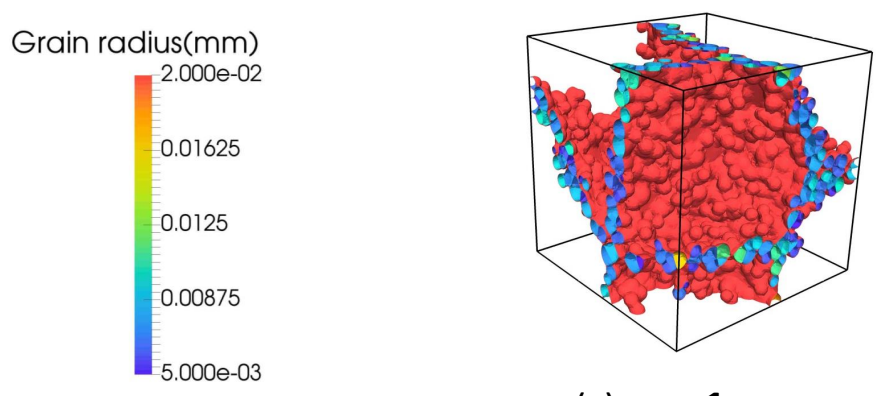

(c) $\varepsilon=1$

Figure 6: Microstructural evolution during a full field simulation of DRX performed at a strain rate of $0.01 \mathrm{~s}^{-1}$ (a) at the initial stage of the simulations (b) at $\varepsilon=1$ with representing the topological deformation of the RVE (c) at $\varepsilon=1$ without representing the topological deformation of the RVE. 


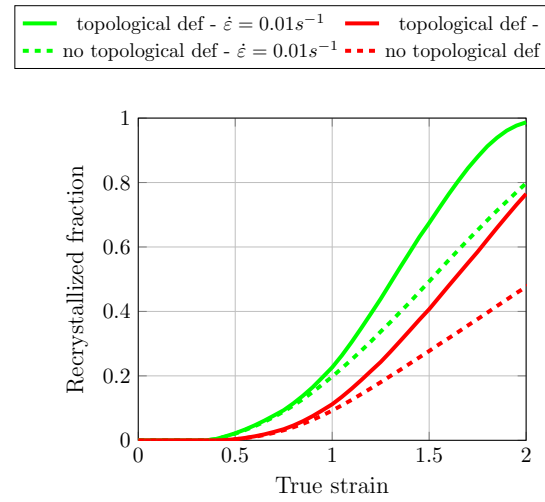

(a)

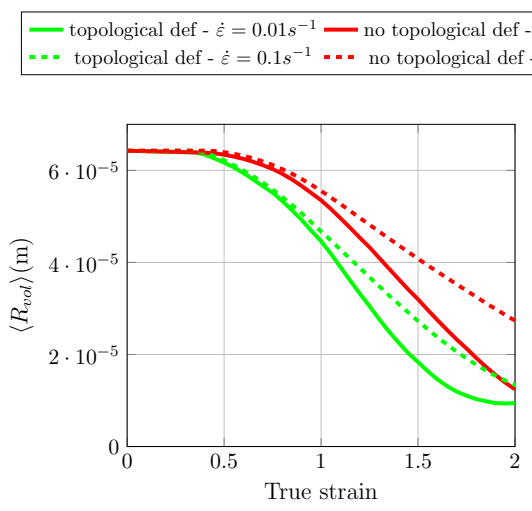

(c)

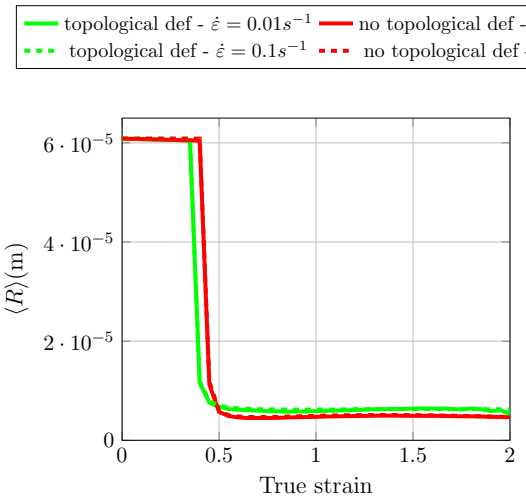

(b)

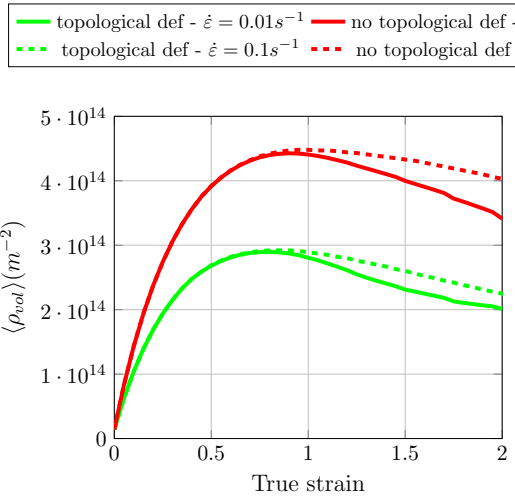

(d)

Figure 7: Comparisons of the results obtained with the full field model described in [19], with and without representing the topological deformation of the RVE. The conditions of deformation are a temperature of $1273 \mathrm{~K}$ and two different strain rates of $0.01 \mathrm{~s}^{-1}$ and $0.1 \mathrm{~s}^{-1}$. The model parameters are those of a $304 \mathrm{~L}$ steel.

The consideration of the topological deformation of the RVE has a clear influence on recrystallization kinetics, mean grain size evolution and mean dislocation density evoRVE is modeled, the grain shape evolves from equiaxed to elongated, which increases the grain boundary surface and thus the nucleation rate (Eq. 77). The following section aims to enrich the NHM by modeling the evolution of principal lengths of the grains (in the sense of idealized ellipsoid shapes) during a dynamic process.

285

In standard mean field modeling of DRX [14, 15, 17], each grain is defined by an equiv- 
alent sphere radius $R_{\mathrm{i}}$ and an average dislocation density $\rho_{\mathrm{i}}$. In this section, grains are assumed to evolve toward an ellipsoidal shape during the deformation process. Thus, for each ellipsoidal grain $i$, a local coordinate system $\left(\overrightarrow{e_{1}^{\mathrm{i}}}, \overrightarrow{e_{2}^{\mathrm{i}}}, \overrightarrow{e_{3}^{\mathrm{i}}}\right)$ that is aligned in the three main directions of the ellipsoid is considered. Three values $(a(\mathrm{i}), b(\mathrm{i}), c(\mathrm{i}))$ represent the three principal semi-axis lengths of the ellipsoidal grain $i$ in its local coordinate system $\left(\overrightarrow{e_{1}^{\mathrm{i}}}, \overrightarrow{e_{2}^{\mathrm{i}}}, \overrightarrow{e_{3}^{\mathrm{i}}}\right)$. Furthermore each grain is associated with an orientation matrix $M_{\mathrm{i}}$ to express the canonical coordinate system $(\vec{x}, \vec{y}, \vec{z})$ in the local coordinate system $\left(\overrightarrow{e_{1}^{\mathrm{i}}}, \overrightarrow{e_{2}^{\mathrm{i}}}, \overrightarrow{e_{3}^{\mathrm{i}}}\right)$. Therefore, each grain $i$ is now defined in the NHM by three semiaxis $(a(\mathrm{i}), b(\mathrm{i}), c(\mathrm{i}))$, a dislocation density $\rho_{\mathrm{i}}$ and a rotation matrix $M_{\mathrm{i}}$. Thus its volume can be deduced by $V_{\mathrm{i}}=(4 / 3) \pi a(\mathrm{i}) b(\mathrm{i}) c(\mathrm{i})$ and its equivalent sphere radius by $R_{\mathrm{i}}=\left[\left(3 V_{\mathrm{i}}\right) /(4 \pi)\right]^{1 / 3}$.

Considering any kind of solicitation, the macroscopic strain tensor $E$ applied on the material in the canonical coordinate system $(\vec{x}, \vec{y}, \vec{z})$ can be written as follows :

$$
\mathbf{E}=\left(\begin{array}{lll}
E_{x x} & E_{x y} & E_{x z} \\
E_{y x} & E_{y y} & E_{y z} \\
E_{z x} & E_{z y} & E_{z z}
\end{array}\right)
$$

Therefore the local strain tensor $L_{\mathrm{i}}$ in the coordinate system of a given ellipsoidal grain $i$ can be obtained by the following relation :

$$
L_{\mathrm{i}}=M_{\mathrm{i}} E M_{\mathrm{i}}^{\mathrm{t}},
$$

The obtained local strain tensor $L_{\mathrm{i}}$ can be written :

$$
\mathbf{L}_{\mathrm{i}}=\left(\begin{array}{lll}
\varepsilon_{11} & \varepsilon_{12} & \varepsilon_{13} \\
\varepsilon_{21} & \varepsilon_{22} & \varepsilon_{23} \\
\varepsilon_{31} & \varepsilon_{32} & \varepsilon_{33}
\end{array}\right)
$$

This local strain tensor can be used in the considered ellipsoidal grain to update its three semi-axis $(a(\mathrm{i}), b(\mathrm{i}), c(\mathrm{i}))$. However the obtained strain tensor $L_{\mathrm{i}}$ is not necessarily 
diagonal and shear strain can be observed. In order to keep the grains ellipsoidal, only the terms in $L_{\mathrm{i}}$ that are in the diagonal are considered. This assumption means that if only shear strain components are observed in the local strain tensor $L_{\mathrm{i}}$ of a grain $i$, its three semi-axis $(a(\mathrm{i}), b(\mathrm{i}), c(\mathrm{i}))$ will not change. If $\varepsilon$ is small enough at each time increment, the evolution of $(a(\mathrm{i}), b(\mathrm{i}), c(\mathrm{i}))$ can be expressed as follows:

$$
\begin{aligned}
& a(\mathrm{i})^{(\mathrm{t}+\Delta \mathrm{t})}=a(\mathrm{i})^{\mathrm{t}}\left(1+\varepsilon_{11}\right), \\
& b(\mathrm{i})^{(\mathrm{t}+\Delta \mathrm{t})}=b(\mathrm{i})^{\mathrm{t}}\left(1+\varepsilon_{22}\right), \\
& c(\mathrm{i})^{(\mathrm{t}+\Delta \mathrm{t})}=c(\mathrm{i})^{\mathrm{t}}\left(1+\varepsilon_{33}\right) .
\end{aligned}
$$

When a grain grows due to boundary migration (Eq.22), its three semi-axis $(a(\mathrm{i}), b(\mathrm{i}), c(\mathrm{i}))$ must also be updated. In this model, growth of a grain is assumed isotropic, thus the three semi-axis $(a(\mathrm{i}), b(\mathrm{i}), c(\mathrm{i}))$ of a grain $i$ having undergone a variation volume are updated following the relations :

$$
\begin{aligned}
& a(\mathrm{i})^{(\mathrm{t}+\Delta \mathrm{t})}=a(\mathrm{i})^{\mathrm{t}} \times^{3} \sqrt{\frac{V_{\mathrm{i}}^{(\mathrm{t}+\Delta \mathrm{t})}}{V_{\mathrm{i}}^{\mathrm{t}}}}, \\
& b(\mathrm{i})^{(\mathrm{t}+\Delta \mathrm{t})}=b(\mathrm{i})^{\mathrm{t}} \times^{3} \sqrt{\frac{V_{\mathrm{i}}^{(\mathrm{t}+\Delta \mathrm{t})}}{V_{\mathrm{i}}^{\mathrm{t}}}}, \\
& c(\mathrm{i})^{(\mathrm{t}+\Delta \mathrm{t})}=c(\mathrm{i})^{\mathrm{t}} \times^{3} \sqrt{\frac{V_{\mathrm{i}}^{(\mathrm{t}+\Delta \mathrm{t})}}{V_{\mathrm{i}}^{\mathrm{t}}}} .
\end{aligned}
$$

where $V_{\mathrm{i}}^{\mathrm{t}}$ and $V_{\mathrm{i}}^{(\mathrm{t}+\Delta \mathrm{t})}$ are the volume of the grain $i$ at the instants $t$ and $(t+\Delta t)$ respectively.

The evolution of principal lengths of a grain $i$ during deformation leads to an increase of its boundary surface. The grain boundary surface of any ellipsoidal grain can be calculated using incomplete elliptic integral of the first and second kind. However to make it simpler and to decrease the computational cost, the surface of a grain $i$ is estimated using the relation [37] :

$$
S_{\mathrm{i}} \simeq 2 \pi\left(a(\mathrm{i})^{\mathrm{p}} b(\mathrm{i})^{\mathrm{p}}+a(\mathrm{i})^{\mathrm{p}} c(\mathrm{i})^{\mathrm{p}}+b(\mathrm{i})^{\mathrm{p}} c(\mathrm{i})^{\mathrm{p}}\right)^{1 / \mathrm{p}},
$$

where $p=\ln (3) / \ln (2)$. This equation gives the surface of a general ellipsoid with a relative error $<1.42 \%$. With this approach, the grain boundary area of all grains will 
increase during deformation and the constitutive equation of nucleation rate Eq. 7 will be affected. This leads to an acceleration of the nucleation kinetics. In standard mean field models in the literature [14, 15, 17], the initial microstructures are composed of spherical grains. Thanks to this new formulation, it is now possible to perform a DRX simulation on an initial microstructure composed of elongated grains, by defining particular initial values of $(a(\mathrm{i}), b(\mathrm{i}), c(\mathrm{i}))$ for each grain $i$.

\section{Comparison with full field results}

In the present section, a recent published full field model of DRX [19] is used to discuss the NHM results. This full field model [19] is based on the same constitutive laws that are used in the present work for strain hardening, recovery and nucleation. The only difference between the full field model [19] and the present NHM is that the microstructure is explicitly described in the full field methodology which means that the comparison between both will indicate if the new microstructure description proposed in the NHM is accurate. An illustrated case of a DRX simulation using the full

340 field model is presented on Fig. 8. The simulated process is a channel-die compression on a $304 \mathrm{~L}$ steel at a temperature of $1273 \mathrm{~K}$ and a strain rate of $0.01 \mathrm{~s}^{-1}$. 


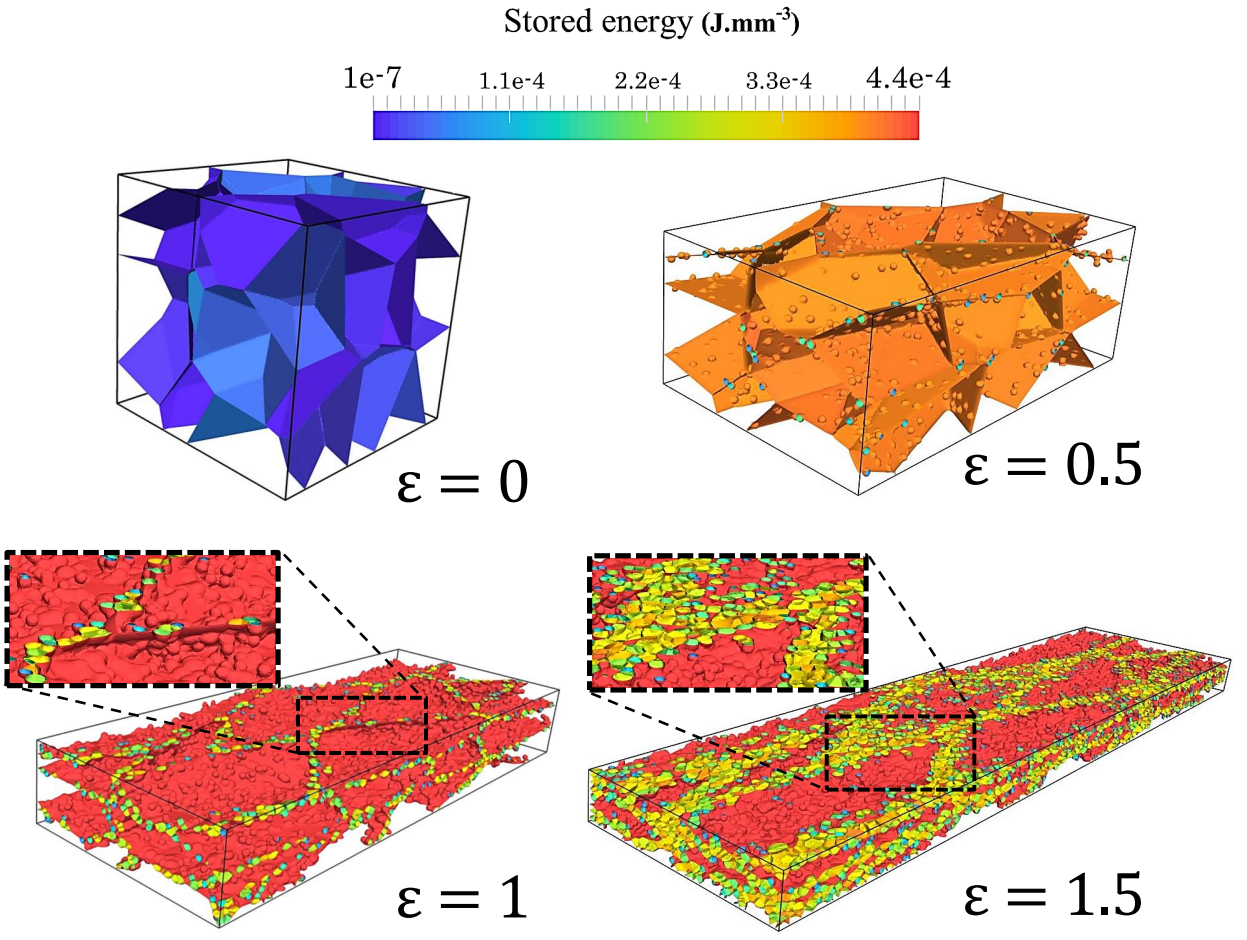

Figure 8: Illustrated case of a DRX simulation using the full field model [19]. The simulated process is a channel-die compression on a $304 \mathrm{~L}$ steel at a temperature of $1273 \mathrm{~K}$ and a strain rate of $0.01 \mathrm{~s}^{-1}$. 


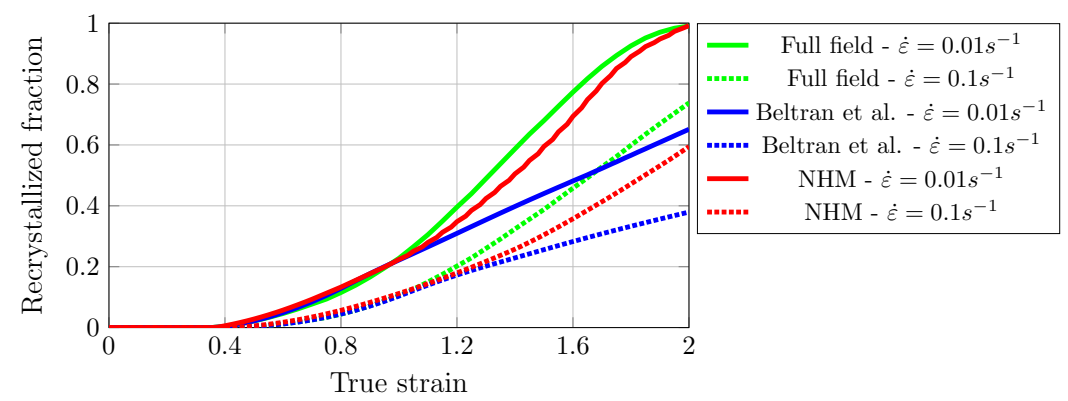

(a)

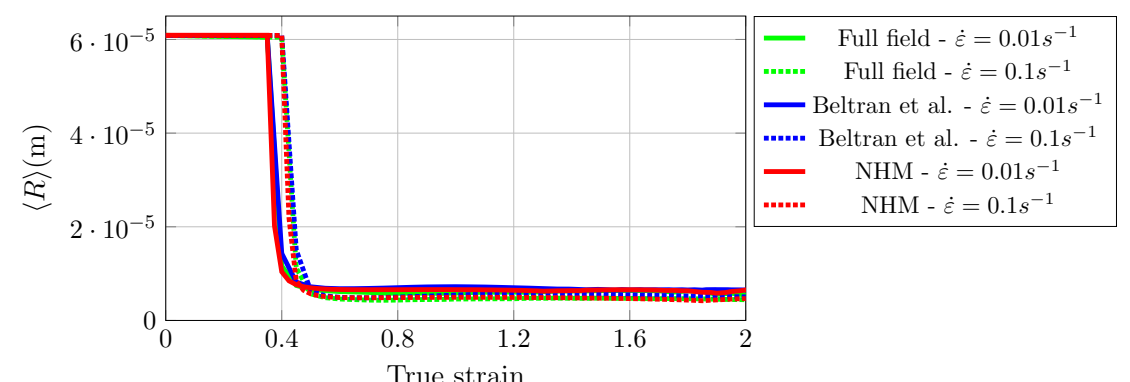

(b)

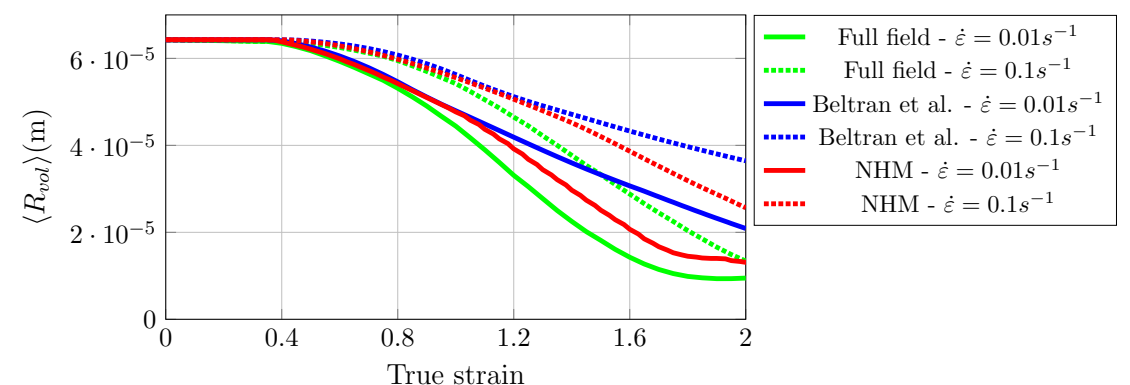

(c)

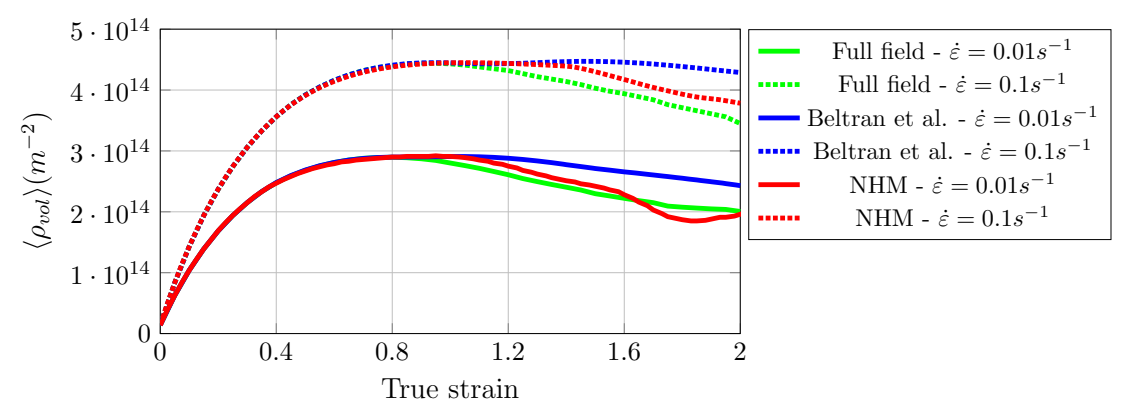

(d)

Figure 9: Comparison of the results obtained by three different models: the full field model [19], the mean field model of Beltran et al. [17] and the NHM proposed in this paper. Compared results are: (a) recrystallized fraction (b) mean grain size, (c) mean grain size weighted by grain volume and (d) mean dislocation density weighted by grain volume. The simulated process is a channel-die compression at $1273 \mathrm{~K}$, at two different strain rates of $0.01 \mathrm{~s}^{-1}$ and $0.1 \mathrm{~s}^{-1}$. 
The results in terms of recrystallized fraction, mean grain radius and mean dislocation density obtained from the mean field model of Beltran [17], the NHM and full field simulations are compared on Fig. 9. The evolution of the recrystallized fraction and mean grain size weighted by volume obtained with the model of Beltran [17] are quasi-linear for the two considered strain rates (see Figs. 9 a) and (c)). With the present NHM, the recrystallized fraction follows an Avrami evolution (i.e. with a "S" shape, see Fig. 9(a)), which is commonly observed in experimental investigations of DRX $[38,39]$. Furthermore, evolutions of recrystallized fraction, mean grain size weighted by volume and dislocation density weighted by volume described with the NHM are quite close to those described by the full field model. Finally, the evolution of the mean grain size (see Fig. 9(b)) is correctly described with both the NHM and mean field model of Beltran [17]. It is also observed that the recrystallization kinetics are always faster in the full field case. This observation can be due to the fact that the surface $S 1$ (i) is approximated by the surface S3(i) in the NHM and model of Beltran [17] with S3(i) $<S 1$ (i) (see Fig. 3), whereas $S 1$ (i) is explicitly taken into account in the full field case. Thus the total boundary surface $\Phi$ considered in the nucleation rate (Eq. 7) is always underestimated in the NHM and model of Beltran [17], leading to slower kinetics of recrystallization.

As already mentioned, a known drawback of pre-existing mean field models if that they provide grain size distributions which do not match with experimental ones. The grain size distributions obtained with the full field model, the NHM and the model of Beltran [17] are compared on Fig. 10 These distributions are compared at four strain levels $\varepsilon=0.5, \varepsilon=1, \varepsilon=1.5$ and $\varepsilon=2$. The grain size distributions described by the NHM are globally close to grain size distributions obtained with the full field model. Furthermore, the grain size distributions obtained with the NHM are quite wide and spread, which is characteristic of experimental distributions observed during DRX [40]. The grain size distributions obtained with the model of Beltran [17] are more tight, which is the characteristic shape observed in the grain size distributions predicted by standard mean field models [14, 15, 17]. This issue has already been discussed in the state of the art [40] and is due to the particularity of Eq. 2 In fact, 
when nuclei appear during the simulation, they growth in the microstructure while increasing their dislocation density by strain hardening. When they reach a given size and dislocation density, they decrease with respect to other nuclei recently appeared. Therefore, the grains cannot growth more than a given size characterized by a high peak on distributions provided by the model of Beltran [17] (see Fig. 10]. 

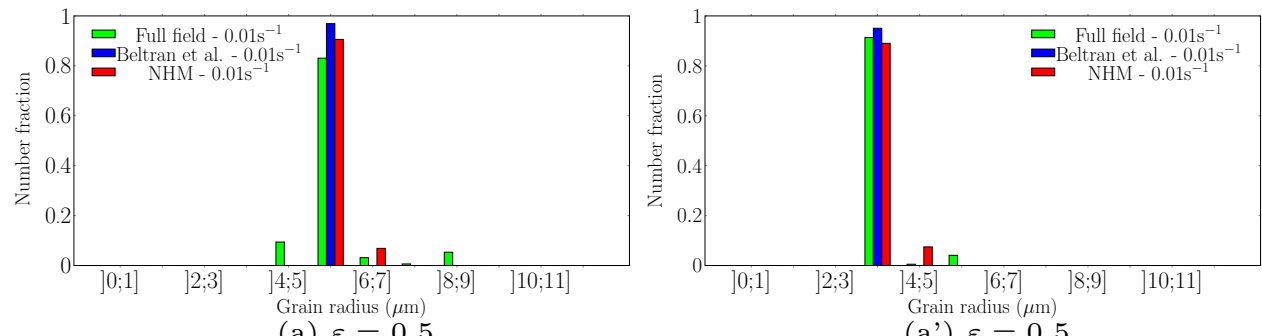

(a) $\varepsilon=0.5$
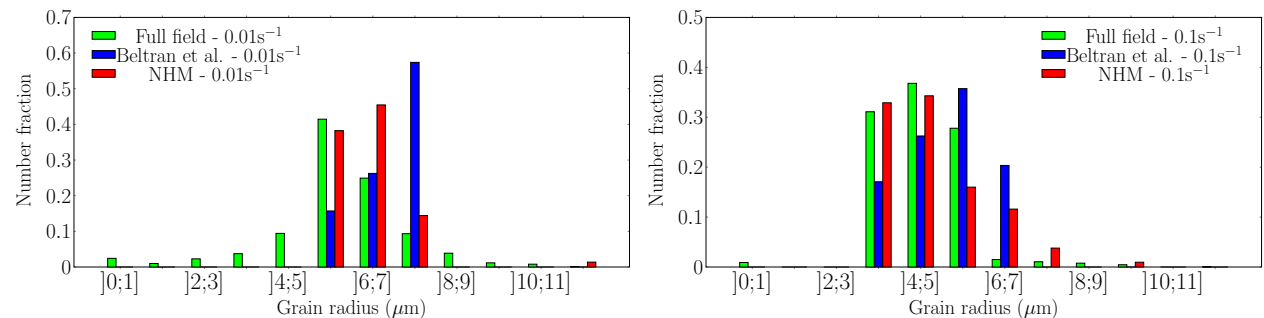

(b) $\varepsilon=1$

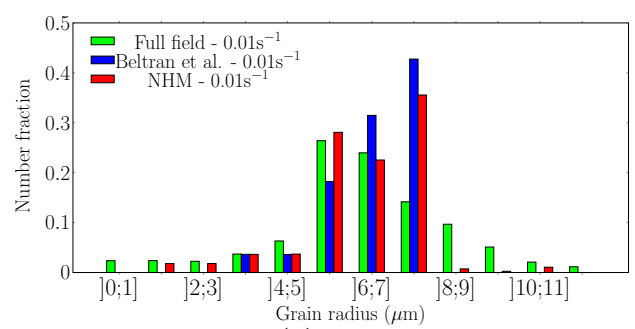

(c) $\varepsilon=1.5$

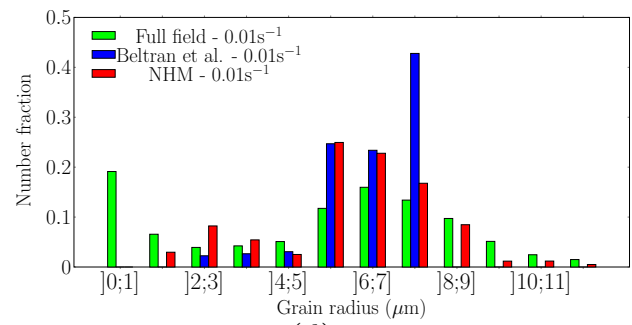

(d) $\varepsilon=2$

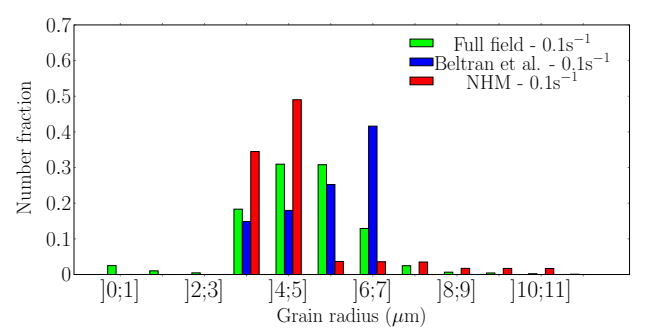

$\left(c^{\prime}\right) \varepsilon=1.5$

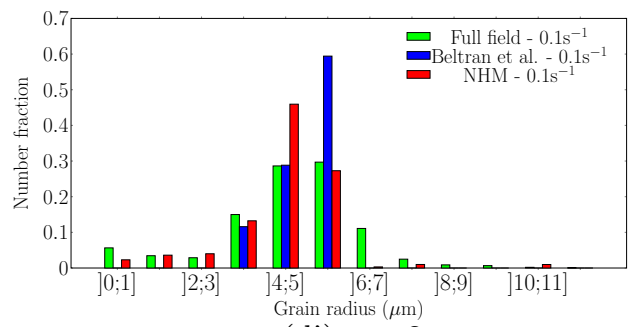

(d') $\varepsilon=2$

Figure 10: Comparison of the grain radius distributions obtained with the full field model [19], the mean field model of Beltran et al. [17] and the NHM. The simulated process is a channel-die compression at 1273K during 200s at two strain rates $0.01 \mathrm{~s}^{-1}$ and $0.1 \mathrm{~s}^{-1}$ and interrupted at different strain levels ((a) to (d)).

As already mentioned, the great advantage of mean field models is their computational cost. This gives them the possibility to be coupled with macroscopic finite element simulations to predict microstructural evolutions throughout the processed product. To 
perform such coupling, many sensors must be placed onto or into the forged part (at each integration points for example) to follow the thermomechanical path (temperature and strain rate as a function of time) at any point of the part during the finite element simulation. Then, a mean field simulation is performed at each sensor using the recorded thermochemical path as input in order to predict the microstructure evolutions. Since a mean field simulation is performed at each sensor, the computational cost of a single simulation is very important to be able to perform a large number of simulations on a relatively short time. The computational cost of a NHM simulation mainly depends on the total number of classes, which is affected by the initial number of classes, the deformation step and the strain level. Therefore, several simulations using the NHM have been performed with three different initial number of classes $(8,16$ and 24 classes) considering the same initial mean grain size. Resulting recrystallized fraction, computational cost, mean grain radius as well as mean dislocation density evolutions are presented on Fig. 11. The obtained results are not really dependant on the initial number of classes (see Fig. 11(a), (c) and (d)). However, the computational cost seems to increase quite linearly with the initial number of classes (see Fig. 11 b)). Thus, a number of 8 initial grain classes is a good compromise between low computational cost and converged results. It is worth mentioning that for this case, the number of classes at the end of the simulation is around 90 . 


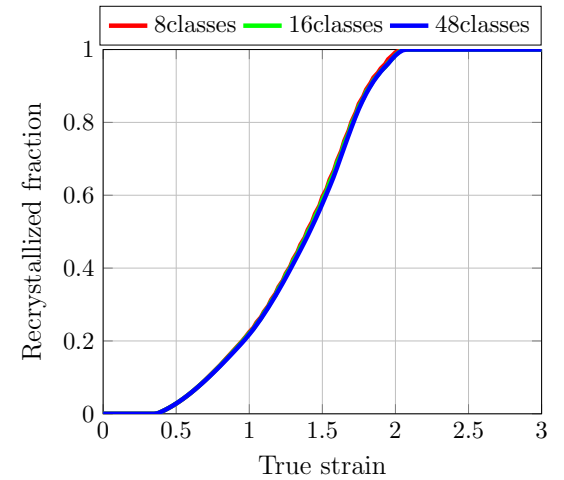

(a)

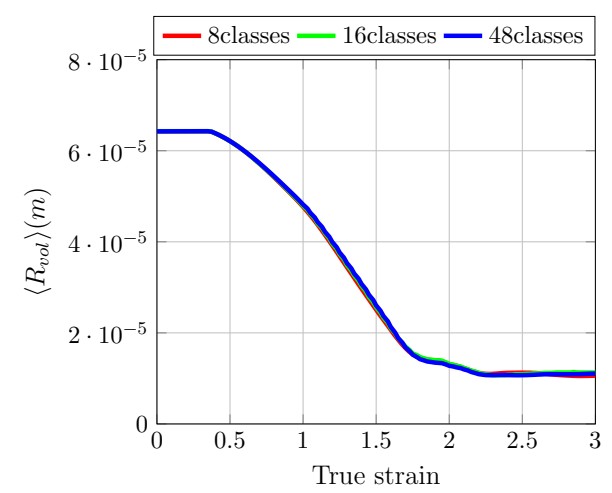

(c)

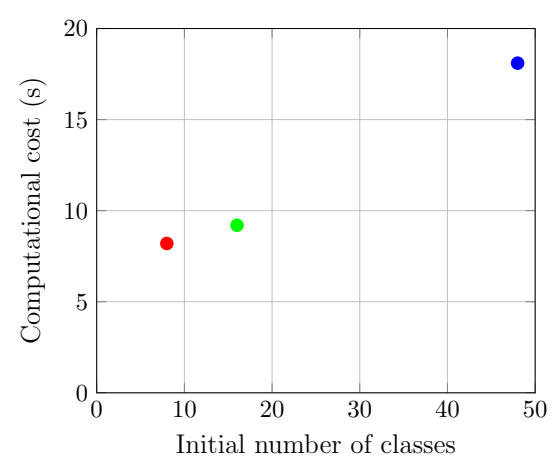

(b)

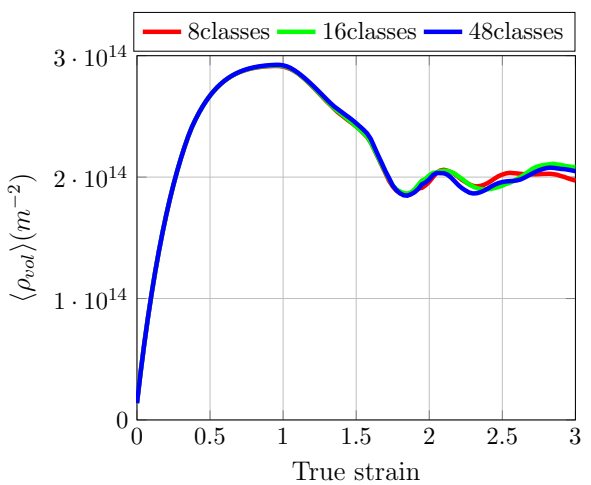

$(d)$

Figure 11: Sensitivity study of the initial number of grain classes on results obtained with the NHM : (a) recrystallized fraction (b) computational cost (c) mean grain radius weighted by grain volume (d) mean dislocation density weighted by grain volume.

Then several simulations using the NHM have been performed with an initial number of 8 grain classes and by considering different deformation steps $(\Delta \varepsilon=0.01, \Delta \varepsilon=$ 0.025 and $\Delta \varepsilon=0.05$ ). Results in terms of recrystallized fraction, computational cost, mean grain radius as well as mean dislocation density are presented on Fig. 12 The recrystallized fraction evolution obtained with the NHM with the three different deformation steps are quite similar (see Fig. 12(a)). However, the final value of the mean dislocation density and mean grain size obtained with the NHM with a deformation step of 0.05 is a bit different from those obtained with a deformation step of 0.01 or 0.025 , the latter being close to each other. It is also observed that the computational 

deformation step of 0.025 leads to computational cost significantly lower than using a deformation step of 0.01 . The influence of the deformation step on the computational cost comes from the fact that the deformation step has a strong influence on the apparition of new classes during the simulation. During nucleation, a new class is created at each deformation step, thus the smaller the deformation step, the more new classes will be created during the simulation. A deformation step of 0.025 is retained as a good compromise between converged results and low computational cost.

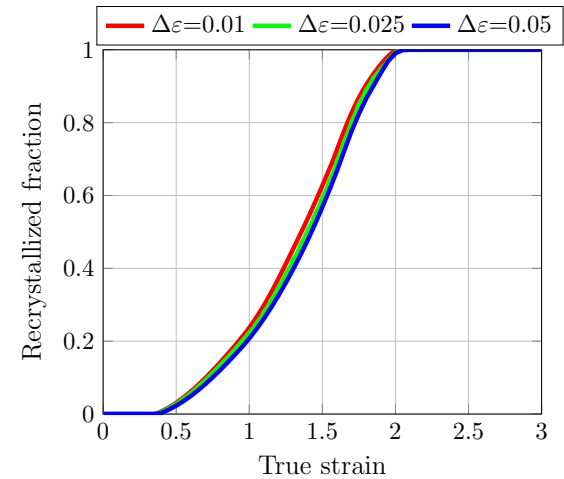

(a)

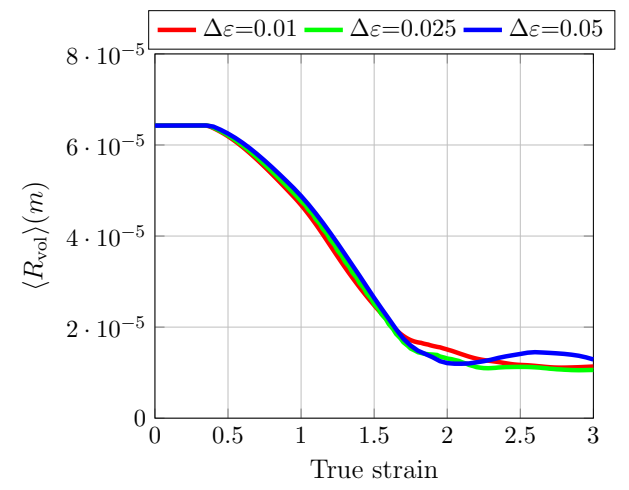

(c)

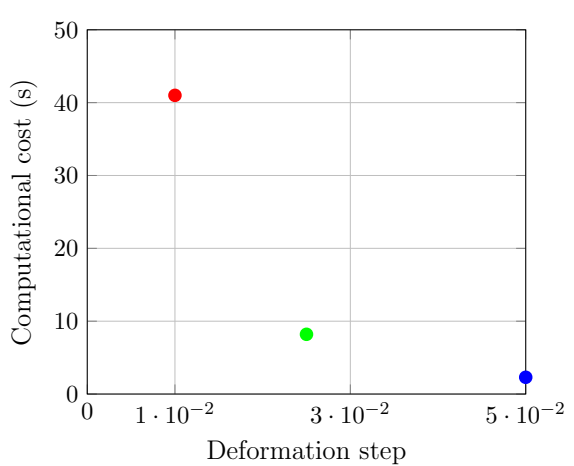

(b)

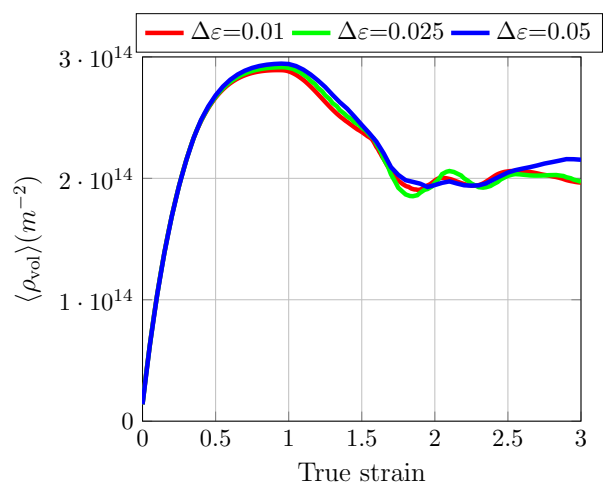

$(d)$

Figure 12: Sensitivity study of the deformation step on results obtained with the NHM : (a) recrystallized fraction (b) computational cost (c) mean grain radius weighted by grain volume (d) mean dislocation density weighted by grain volume.

Finally, since the computational cost also depends on the strain level, the Fig. 13 
presents the evolution of the number of increments as well as the cumulated computational cost as a function of the true strain during a simulation using the NHM. A number of 8 initial classes as well as a deformation step of 0.025 were considered in the simulation. First the evolution of the number of increments is linear up to a strain level of 1.2 and then evolves faster. This increase is due to the strategy adopted in the model of Beltran [17] and used in the NHM. The latter consists in adapting the deformation step in order to avoid grains with a negative volume, which can appear with Eq. 2 or 17 This adaptation means that each deformation step can be discretized in several increment during the simulation. The cumulated computational cost also increases as a function of the true strain during the simulation. This evolution is slow up to a strain level of 1.2 since the number of classes is still low at the beginning of the 430 simulation. Then the evolution of the computation cost increases faster up to the end of the simulation. This increases is due to the fact that the number of increments as well as the number of classes are constantly increasing all along the simulation. The global computational cost of the NHM simulation is still very low even at high level of strain. Therefore if a finite element simulation of hot forging is composed of 50.000 integration, reaching a homogeneous strain level of 2 , it is possible to simulate the microstructural evolutions on the entire forged part in less than one week using the NHM, which would have taken almost few years using a full field model. 


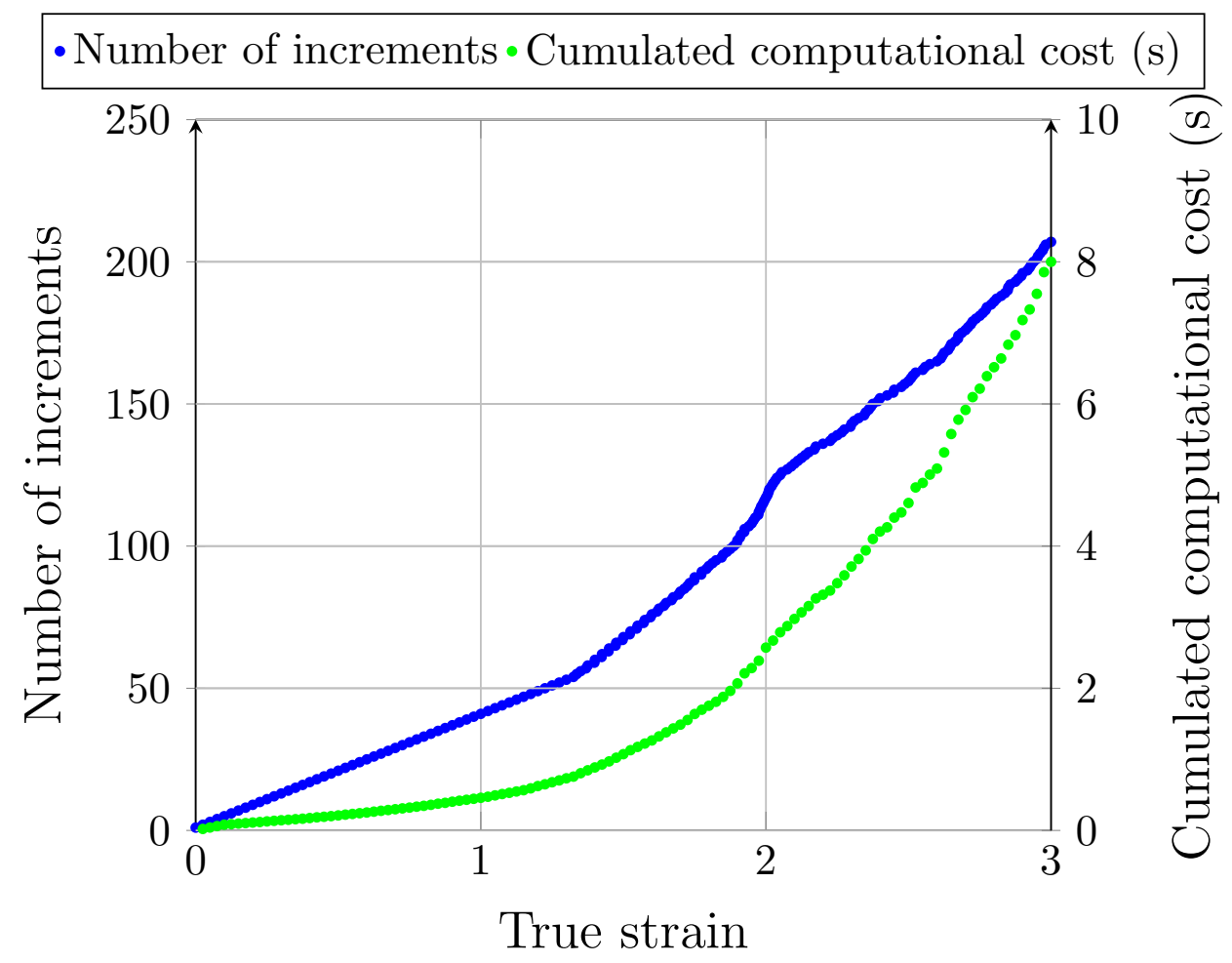

Figure 13: Number of increment and cumulated computation cost as a function of the true strain obtained during a simulation using the NHM at a strain rate of $0.01 \mathrm{~s}^{-1}$ and a temperature of $1273 \mathrm{~K}$. A number of 8 initial grain classes and a deformation step of 0.025 were considered.

\section{Conclusion}

A new topological approach called "NHM" for NeighborHood Model is proposed for the mean field modeling of dynamic recrystallization. The latter is based on two main improvements as compared to the state of art : (i) the consideration of a particular neighborhood for each grain and (ii) the modeling of principal lengths of grains. The results obtained by NHM are compared to those obtained by a former mean field model [17] and non-negligible improvements are observed in terms of average quantities as well as grain size distributions. A sensitivity study showed that the NHM is interesting in terms of numerical cost, which gives the opportunity to simulate the microstructural evolutions on an entire forged part issued from a finite element calculation in less than 
one week. Despite large advances in terms of predictions as compared to former mean field formulations, some improvements concerning neighbors choices can still be done based on experimental investigations. Furthermore, an extension of NHM for postdynamic and static evolutions is required to simulate multi-pass deformations.

\section{Acknowledgements}

The authors thank the AREVA NP, ArcelorMittal, ASCOMETAL, AUBERT \& DUVAL, CEA, SAFRAN, TIMET, Constellium and TRANSVALOR companies and the ANR for their financial support through the DIGIMU consortium and ANR industrial Chair.

\section{References}

[ [1] D. Li, Q. Guo, S. Guo, H. Peng, Z. Wu, The microstructure evolution and nu1. cleation mechanisms of dynamic recrystallization in hot-deformed Inconel 625

460 superalloy, Materials and Design 32 (2) (2011) 696-705. doi:10.1016/j. matdes.2010.07.040.

URL http://dx.doi.org/10.1016/j.matdes.2010.07.040

口[2] H. Jiang, L. Yang, J. Dong, M. Zhang, Z. Yao, The recrystallization model and microstructure prediction of alloy 690 during hot deformation, Materials and Design 104 (2016) 162-173. doi:10.1016/j.matdes.2016.05.033

URL http://dx.doi.org/10.1016/j.matdes.2016.05.033

口 [3] Z. Wan, Y. Sun, L. Hu, H. Yu, Experimental study and numerical simulation of dynamic recrystallization behavior of TiAl-based alloy, Materials \& Design 122 (2017) 11-20. doi:10.1016/j.matdes.2017.02.088.

q [4] K. Huang, R. Logé, A review on dynamic recrystallization phenomena in metallic materials, Materials \& Design 111 (2016) 548-574. doi:10.1016/j.matdes.2016.09.012 


I URL http://linkinghub.elsevier.com/retrieve/pii/
S0264127516311753

[ [5] H. Abedi, A. Zarei Hanzaki, Z. Liu, R. Xin, N. Haghdadi, P. Hodgson, Continuous dynamic recrystallization in low density steel Materials \& Design 114 (2017) 55-64. doi:10.1016/j.matdes.2016.10.044.

a URL http://linkinghub.elsevier.com/retrieve/pii/ S0264127516313429

[6] A. Kolmogorov, On the Statistical Theory of Crystallization of Metals, Izv. Akad. Nauk SSSR, Ser. Mat. 3 (1937) 355-359.

[7] W. Johnson, R. Mehl, Reaction Kinetics in Processes of Nucleation and Growth, Transactions of the American Institute of Mining and Metallurgical Engineers 135 (1939) 416-442.

[8] M. Avrami, Kinetics of Phase Change. I General Theory, The Journal of Chemical Physics 7 (12) (1939) 1103-1112. doi:10.1063/1.1750380. URL http://aip.scitation.org/doi/10.1063/1.1750380

${ }_{4}$ [9] C. E. Krill, L. Q. Chen, Computer simulation of 3-D grain growth using a phase-field model Acta Materialia 50 (12) (2002) 3059-3075. doi:10.1016/S1359-6454(02)00084-8. URL http://wWw.sciencedirect.com/science/article/ B6TW8-466R5TY-1/2/4e83847503c9ca5646b71d4de608f2fa

[10] M. Bernacki, Y. Chastel, T. Coupez, R. Logé, Level set framework 495 for the numerical modelling of primary recrystallization in polycrystalline materials, Scripta Materialia 58 (12) (2008) 1129-1132. doi:10.1016/j.scriptamat.2008.02.016.

1 URL http://linkinghub.elsevier.com/retrieve/pii/ S1359646208001425

500 [11] A. D. Rollett, D. Raabe, A hybrid model for mesoscopic simulation of recrystallization, Computational Materials Science 21 (1) (2001) 69-78. 
doi:10.1016/S0927-0256(00)00216-0.

URL http://www.sciencedirect.com/science/article/pii/ S0927025600002160

[12] D. Raabe, Introduction of a scalable three-dimensional cellular automaton with a probabilistic switching rule for the discrete mesoscale simulation of recrystallization phenomena, Philosophical Magazine A 79 (10) (1999) 2339-2358. doi:10.1080/01418619908214288.

[1 URL http://www.tandfonline.com/doi/abs/10.1080/ 01418619908214288

[13] M. Hillert, On the theory of normal and abnormal grain growth Acta Metallurgica 13 (3) (1965) 227-238. doi:10.1016/0001-6160(65)90200-2.

10 URL http://www.sciencedirect.com/science/article/pii/ 0001616065902002?via\{\%\}3Dihub

515 [14] F. Montheillet, O. Lurdos, G. Damamme, A grain scale approach for modeling steady-state discontinuous dynamic recrystallization, Acta Materialia 57 (5) (2009) 1602-1612. doi:10.1016/j.actamat.2008.11.044 URL http://linkinghub.elsevier.com/retrieve/pii/ S1359645408008641

[15] D. G. Cram, H. S. Zurob, Y. J. M. Brechet, C. R. Hutchinson, Modelling discontinuous dynamic recrystallization using a physically based model for nucleation, Acta Materialia 57 (17) (2009) 5218-5228. doi:10.1016/j .actamat.2009. 07.024

URL http://dx.doi.org/10.1016/j . actamat.2009.07.024

${ }_{525}[16]$ P. Bernard, S. Bag, K. Huang, R. Logé, A two-site mean field model of discontinuous dynamic recrystallization, Materials Science and Engineering: A 528 (24) (2011) 7357-7367. doi:10.1016/j.msea.2011.06.023

1 URL http://linkinghub.elsevier.com/retrieve/pii/ S0921509311006757 
[18] D. Piot, G. Smagghe, F. Montheillet, G. Kermouche, M. Bernacki, A. Montouchet, G. Perrin, Une approche semitopologique pour la modélisation en champs moyens des recristallisations dynamique et métadynamique, in: Journées annuelles SF2M, Lyon, France, 2017.

540

URL

https://hal-mines-paristech.archives-ouvertes.fr/ hal-01625123

[19] L. Maire, B. Scholtes, C. Moussa, N. Bozzolo, D. Pino Muñoz, A. Settefrati,

[20] G. Abbruzzese, I. Heckelmann, K. Lücke, Statistical theory of two-dimensional grain growthI. The topological foundation Acta Metallurgica et Materialia 40 (3) (1992) 519-532. doi:10.1016/0956-7151(92)90401-Y URL http://www.sciencedirect.com/science/article/pii/ $095671519290401 \mathrm{Y}$

[21] M. Zouari, N. Bozzolo, R. E. Loge, Mean field modelling of dynamic and 【 post-dynamic recrystallization during hot deformation of Inconel 718 in the absence of $\delta$ phase particles Materials Science and Engineering: A 655 (2016) 1-17. doi:10.1016/j.msea.2015.12.102. 


aRL http://linkinghub.elsevier.com/retrieve/pii/
S0921509315307930

[22] L. Maire, B. Scholtes, C. Moussa, N. Bozzolo, D. Pino Muñoz, M. Bernacki, 1. Improvement of 3D mean field models for capillarity-driven grain growth based on full field simulations, Journal of Materials Science 51 (24) (2016) 1097010981. doi:10.1007/s10853-016-0309-6. URL http://link.springer.com/10.1007/s10853-016-0309-6

[23] A. Yoshie, H. Morikawa, Y. Onoe, K. Itoh, Formulation of static recrystallization of austenite in hot rolling process of steel plate., Transactions of the Iron and Steel Institute of Japan 27 (6) (1987) 425-431. doi:10.2355/isijinternational1966.27.425. URL http://joi.jlc.jst.go.jp/JST.Journalarchive/ isijinternational1966/27.425?from=CrossRef

[24] J. E. Bailey, P. B. Hirsch, The Recrystallization Process in Some Polycrystalline Metals, Proceedings of the Royal Society A: Mathematical, Physical and Engineering Sciences 267 (1328) (1962) 11-30. doi:10.1098/rspa.1962.0080

575 URL http://rspa.royalsocietypublishing.org/cgi/doi/10.1098/ rspa.1962.0080

[25] P. Peczak, M. J. Luton, The effect of nucleation models on dynamic recrystallization I. Homogeneous stored energy distribution, Philosophical Magazine Part B 68 (1) (1993) 115-144. doi:10.1080/13642819308215285.

580 URL http://www.tandfonline.com/doi/abs/10.1080/ 13642819308215285

[26] S.-I. Kim, B.-C. Ko, C.-M. Lee, S.-K. Hwang, Y.-C. Yoo, Evolution of dynamic recrystallisation in AISI 304 stainless steel. Materials Science and Technology 19 (12) (2003) 1648-1652. doi:10.1179/026708303225008284 585 URL http://www.tandfonline.com/doi/full/10.1179/ 026708303225008284 
[27] L. Gavard, F. Montheillet, Dynamic recrystallization and grain refinement in a high purity 304L type austenitic stainless steel. Matériaux \& Techniques 88 (5-6) (2000) 65-68. doi:10.1051/mattech/200088050065. 200088050065

[28] R. T. DeHoff, G. Q. Liu, On the relation between grain size and grain topol-

1 ogy, Metallurgical Transactions A 16 (11) (1985) 2007-2011. doi:10.1007/ BF02662401. URL http://link . springer . com/10.1007/BF02662401

[29] G. Liu, H. Yu, X. Qin, Three-dimensional grain topologysize relationn. ships in a real metallic polycrystal compared with theoretical models. Materials Science and Engineering: A 326 (2) (2002) 276-281. doi:10.1016/S0921-5093(01)01497-6 URL http://www.sciencedirect.com/science/article/pii/ S0921509301014976

[30] G. Abbruzzese, A. Campopiano, Topological aspects of grain growth microstructure: The two-dimensional and three-dimensional cases., in: STERMAT94, Proceedings of the 4th International Conference on Stereology and Image Analysis in Material Science, Wisla, Poland, 1994.

[31] C. Zhang, M. Enomoto, A. Suzuki, T. Ishimaru, Characterization of threedimensional grain structure in polycrystalline iron by serial sectioning, Metallurgical and Materials Transactions A 35 (7) (2004) 1927-1933. doi:10.1007/ s11661-004-0141-5

URL http://link.springer.com/10.1007/s11661-004-0141-5

[32] S. O. Poulsen, P. W. Voorhees, E. M. Lauridsen, Three-dimensional simu1. lations of microstructural evolution in polycrystalline dual-phase materials with constant volume fractions, Acta Materialia 61 (2013) 1220-1228. doi: $10.1016 / j$.actamat.2012.10.032

615 प URL http://ac.els-cdn.com/S1359645412007793/ 
1-s2.0-S1359645412007793-main.pdf?\{_\}tid=

2de02bb0-6081-11e7-8c65-00000aab0f26 $\{\&\} a c d n a t=$

1499149539\{_\}c422c7c4eb947ea4aeabe29bf9883cd0

[33] D. Zöllner, P. Streitenberger, Normal Grain Growth: Monte Carlo Potts Model

978-3-540-85715-0\{\_\}1

URL http://link.springer.com/10.1007/978-3-540-85715-0\{_\}1

[34] F. Wakai, N. Enomoto, H. Ogawa, Three-dimensional microstructural evolution

[35] B. Scholtes, M. Shakoor, N. Bozzolo, P.-O. Bouchard, A. Settefrati, M. Bernacki, 630

口

[37] G. P. Michon, Final answers (2004) See Thomsen's formulas and Cantrell's comments.

URL http: //wwW.numericana.com/answer/ellipsoid.htm

[38] G. Shen, S. L. Semiatin, R. Shivpuri, Modeling microstructural development during the forging of Waspaloy, Metallurgical and Materials Transactions A 26 (7) 
(1995) 1795-1803. doi:10.1007/BF02670767.

URL http://link.springer.com/10.1007/BF02670767

[39] M. Zouari, R. E. Loge, O. Beltran, S. Rousselle, N. Bozzolo, Multipass forging

1 of Inconel 718 in the delta-Supersolvus domain: assessing and modeling microstructure evolution, in: EUROSUPERALLOYS, Giens, France, 2014. doi:10.1051/matecconf/20141412001

口 URL http://www.matec-conferences.org/10.1051/matecconf/ 20141412001

[40] G. Smagghe, D. Piot, F. Montheillet, A. Montouchet, M. Bernacki, J. Jonas, G. Kermouche, An extended mean field approach for modelling realistic grain size distribution evolutions during Discontinuous Dynamic RX and Post-Dynamic RX, in: 6th International Conference on Recrystallization and Grain Growth: Recrystallization II: Dynamic Recrystallization, Pittsburgh, Pennsylvania, USA, 2016.

a URL https://hal-mines-paristech.archives-ouvertes.fr/ 